\title{
Navier-Stokes Equation and its Fractional Approximations
}

\author{
Tomasz Dlotko 1
}

(C) The Author(s) 2016. This article is published with open access at Springerlink.com

\begin{abstract}
We consider the Navier-Stokes equation (N-S) in dimensions two and three as limits of the fractional approximations. In 2-D the N-S problem is critical with respect to the standard $L^{2}$ a priori estimates and we consider its regular approximations with the fractional power operator $(-P \Delta)^{1+\alpha}, \alpha>0$ small, where $P$ is the projector on the space of divergence-free functions. In 3-D different properties of the N-S problem with respect to the standard $L^{2}$ a priori estimate are obtained and the 3$\mathrm{D}$ regular approximating problem involves fractional power operator $(-P \Delta)^{s}$ with $s>\frac{5}{4}$. Using Dan Henry's semigroup approach and the Giga-Miyakawa estimates we construct regular solutions to such approximations. The solutions are global in time, unique, smooth and regularized through the equation in time. Solution to 2-D and 3-D N-S equations are obtained next as a limit of such regular solutions of the approximations. Moreover, since the nonlinearity of the $\mathrm{N}-\mathrm{S}$ equation is of quadratic type, the solutions corresponding to small initial data and small $f$ are shown to be global in time and regular.
\end{abstract}

Keywords 3-D Navier-Stokes equation · Solvability · A priori estimates · Fractional approximations

Mathematics Subject Classification $35 \mathrm{Q} 30 \cdot 76 \mathrm{D} 05 \cdot 35 \mathrm{~S} 15 \cdot 35 \mathrm{~A} 25$

Dedicated to my Professors: Andrzej Lasota ${ }^{\dagger}$, Jack K. Hale ${ }^{\dagger}$ and Nick D. Alikakos.

Tomasz Dlotko

tdlotko@math.us.edu.pl

1 Institute of Mathematics, Silesian University, Bankowa 14, 40-007 Katowice, Poland 


\section{Introduction}

Fifteen years ago, in the monograph [3], we were studying a direct generalization of semilinear parabolic equations, namely abstract semilinear equation with sectorial positive operator in the main part. Following the idea of Dan Henry [21], such equation will be studied using classical techniques of the theory of ordinary differential equations, modified however to cover equations with unbounded operator in a Banach space. Such approach, located inside the semigroup theory, proves its strength and utility in the study of several classical problems; some of them were reported in $[3,21]$. But such technique offers further possible generalizations, first to study the problems, like e.g. Korteweg-de Vries equation and its extensions [7,8,12,13], where the solutions are obtained as a limit of solutions to parabolic regularizations of such equations (the method known as vanishing viscosity technique, originated by Hopf, Oleinik, Lax in 1950th); see also [9,10]. Another possible application of Henry's technique is to study critical problems (e.g. [3,41]), not falling directly into the class of semilinear sectorial equations because the nonlinear term in it is of the same order, or value, as the main part operator. A recent paper [11] was devoted to such type problem, the quasi-geostrophic equation in $\mathbb{R}^{2}$ (e.g. [4-6,26,44,45]). But certainly the most celebrated example of such critical problem is the Navier-Stokes equation in dimension two. We will obtain and study its solutions constructed as limits of solutions to sub-critical approximations (1.2) when $\alpha \rightarrow 0^{+}$where, in 2-D, the $P \Delta$ operator is replaced with its fractional power $-(-P \Delta)^{1+\alpha}, \alpha>0(P$ is the projector on the space of divergence-free functions; see e.g. [15]). In 3-D a higher order diffusion term like $-(-P \Delta)^{s}, s>\frac{5}{4}$, is considered in the corresponding regularization of the N-S equation because the properties of the problem with respect to the natural $L^{2}$ estimate in that case are different.

The classical 3-D Navier-Stokes equation (N-S) considered here has the form:

$$
\begin{aligned}
u_{t} & =v \Delta u-\nabla p-(u \cdot \nabla) u+f, \quad \text { divu }=0, \quad x \in \Omega, t>0, \\
u & =0, \quad t>0, \quad x \in \partial \Omega, \\
u(0, x) & =u_{0}(x),
\end{aligned}
$$

where $v>0$ is the viscosity coefficient, $u=\left(u_{1}(t, x), u_{2}(t, x), u_{3}(t, x)\right)$ denotes velocity, $p=p(t, x)$ pressure, and $f=\left(f_{1}(x), f_{2}(x), f_{3}(x)\right)$ external force, and $\Omega$ is a bounded domain with $C^{2}$ boundary. It is impossible to recall even the most important results devoted to that problem, since the corresponding literature is too large; see anyway $[2,14-17,20,23,24,29-31,36-38,40]$ together with the references cited there. However, we will try to recall the most important steeps in the semigroup approach to the Navier-Stokes problem, the approach used in the present paper. In 1962 and 1964, thirty years after the Leray fundamental paper [31], the semigroup treatment of the 2-D and 3-D N-S was stated by Fujita and Kato [16,25]. Existence and uniqueness of strong solutions was obtained by solving the evolution equation in $X_{2}$ space, together with basic semigroup formulation and tools. That idea was extended later by Giga and Miyakawa in a series of papers including $[17,18,20]$ in early 1980th. Detail informations concerning analyticity of the semigroup generated by the Stokes 
operator with Dirichlet boundary condition on general $L^{r}$ type spaces, deep estimates of the nonlinearity (including (1.15), below), existence-uniqueness theory in fractional order spaces $D\left(A^{\gamma}\right)$ were reported in that references. Here also the papers $[23,43]$ should be mentioned, contributing to the latter results. Finally, the 1985 monograph by von Wahl, collects and extends in the direction of the global in time solvability and regularity, the existing semigroup results concerning the N-S equation. Meanwhile, in 1981 (with first version in 1974), an abstract approach to semilinear equations with sectorial operators was presented by Dan Henry in [21]. However, the N-S equation is only mentioned there. Finally, an extended discussion of the semigroup approach to the Navier-Stokes equation can be found in the review article [19].

Let describe briefly the contents of the present paper. In space dimensions 2 and 3 the N-S equation possess local in time regular solutions. Using strongly the technique of [20], we recall local in time solvability in Theorems 1.5 and 1.6. We analyze further criticality of the Navier-Stokes equation (compare [3,40,41]), in a sense that available for it $L^{2}(\Omega)$ a priori estimate (1.11) is not sufficient to control its nonlinearity through the viscosity term $v P \Delta u$. Consequently, possible is a balance between the income from nonlinearity and the stabilizing action of the viscosity so that the local solutions can not be extended globally in time. For small initial data the decisive role is played by viscosity, while for larger initial data the nonlinear term is strong enough to unable us to obtain regularization of the solutions through the main part operator. We will try to see such effect through the estimates obtained in the paper.

In 2-D, our idea is to improving a bit the viscosity term to make the whole problem sub-critical, such that the improved viscosity together with the known $L^{2}(\Omega)$ a priori estimate will control the nonlinear term. The way for obtaining such effect is to replace the classical viscosity term $A u=-v P \Delta u$ through a bit higher fractional diffusion $-A^{1+\alpha} u$, with small $\alpha>0$. Next, we will study the process of letting $\alpha$ to $0^{+}$; which properties/estimates of the solutions of regularized problems (with $\alpha>0$ ) are lost in such a limit. We also look at 3-D N-S equation in which case a stronger diffusion term, with $-P \Delta$ operator in the power $s>\frac{5}{4}$, will be considered to guarantee together with the standard $L^{2}(\Omega)$ a priori estimate the control on the nonlinear term.

In 2-D, instead of (1.1), we consider a family of sub-critical problems, with $\alpha \in$ $\left(0, \frac{1}{2}\right]$ :

$$
\begin{aligned}
u_{t} & =-A^{1+\alpha} u-P(u \cdot \nabla) u+P f, \quad t>0, \\
u(0, x) & =u_{0}(x) .
\end{aligned}
$$

The approximation proposed in 3-D is given in (2.6) with $s>\frac{5}{4}$.

Until very recently, equations with fractional power operators were not seriously studied in the literature, therefore we hope the regularizations proposed will help to understand the difficulties faced in the original N-S equation. An analogous phenomena was studied recently [11] for the quasi-geostrophic equation in $R^{2}$ (e.g. $[4-6,44,45])$. The technique used in the present paper is similar to that of [11].

There were several tries of replacing the classical N-S equation, or the viscosity term in it, with another equation having better properties of solutions, starting with Leray $\alpha$-regularization reported in paper [31], see also [14]. Modification of one factor in 
nonlinearity, using mollifier, was sufficient to improve properties of solutions. Another modification of the N-S equation was proposed by Lions in [33, Chapter 1, Remarque 6.11] (see also [30]), where the $-\Delta$ operator was replaced with $-\Delta+\kappa(-\Delta)^{l}, l \geq \frac{5}{4}$. Further modifications can be easily found in the literature, in particular in the closing part of [29]. In fact, in this paper we follow the idea of Lions to replace the diffusion term with a stronger one fractal diffusion term.

\subsection{Introductory Facts}

Notation We are using standard notation for Sobolev spaces. Compare [39] or [3, Chapter 1] for properties of fractional order Sobolev spaces; see also [22] for Sobolev type embeddings. For $r \in \mathbb{R}$, let $r^{-}$denotes a number strictly less than $r$ but close to it. Similarly, $r^{+}>r$ and $r^{+}$close to $r$. When needed for clarity of the presentation, we mark the dependence of the solution $u$ of (1.2) on $\alpha \in\left(0, \frac{1}{2}\right]$, calling it $u^{\alpha}$, or $u^{\epsilon}$ for the approximation in 3-D. Throughout the text the 'standard' or 'natural' a priori estimates mean the estimates (1.11) and (1.12).

Recall first [3, Chapter 3], that studying in a Banach space $X$ an abstract Cauchy's problem with sectorial positive operator $A$ and solutions varying in the phase space $X^{\beta}=D\left(A^{\beta}\right)$ :

$$
\begin{aligned}
u_{t}+A u & =F(u), \\
u(0) & =u_{0},
\end{aligned}
$$

knowing an a priori estimate of all its potential $X^{\beta}$ solutions; $\|u(t)\|_{Y} \leq$ const in another Banach space $D(A) \subset Y$, we say that the nonlinear term $F$ is sub-critical relative to such a priori bound, if for each such $X^{\beta}$ solution $u(t)$ an estimate is valid

$$
\exists_{\theta \in(0,1)} \exists_{\text {nondecreasing }:[0, \infty) \rightarrow[0, \infty)} \quad \| F\left(u(t) \|_{X} \leq g\left(\|u(t)\|_{Y}\right)\left(1+\|u(t)\|_{X^{\beta}}^{\theta}\right),\right.
$$

for all $t \in\left(0, \tau_{u_{0}}\right)$, where $\tau_{u_{0}}$ is the 'life time' of that solution. In that case (e.g. [3, Chapter 3]) the $X^{\beta}$ norm of the local solution will be bounded on $\left[0, \tau_{u_{0}}\right)$, which allows to extend such solution globally in time. Similar extendibility property holds even if $\theta=1$ provided that $\beta<1$ (as we assume above), which in turn is unknown if $\theta=\beta=1$; the nonlinearity $F$ being in the latter case called critical relative to that $a$ priori estimate.

Note that critical nonlinearities are 'of the same order' in the equation as the main part operator $A$ (compare $[40,41]$ ). The main part operator $A$ will not control the nonlinearity in that case, unless we find a better a priori estimate.

\subsection{Properties of the Stokes Operator}

Familiar in the theory of the N-S equation are the following spaces: 


$$
\begin{aligned}
\mathcal{L}^{r}(\Omega) & =\left[L^{r}(\Omega)\right]^{3}, \\
\mathcal{W}^{2, r}(\Omega) & =\left[W^{2, r}(\Omega)\right]^{3}, \\
X_{r} & =\operatorname{cl}_{\mathcal{L}^{r}(\Omega)}\left\{\phi \in\left[C_{0}^{\infty}(\Omega)\right]^{3} ; \operatorname{div} \phi=0\right\},
\end{aligned}
$$

$1<r<\infty$. We define also the Stokes operator $(N=3)$

$$
A_{r}=-v P_{r}\left[\begin{array}{ccc}
\Delta & 0 & 0 \\
0 & \Delta & 0 \\
0 & 0 & \Delta
\end{array}\right]
$$

where $P_{r}$ denotes the projection from $\mathcal{L}^{r}(\Omega)$ to $X_{r}$ given by the decomposition of $\mathcal{L}^{r}(\Omega)$ onto the space of divergence-free vector fields and scalar-function gradient (e.g. [37]). It is further known [20, Lemma1.1], that

Proposition 1.1 The operator $-A_{r}$ considered with the domain

$$
D\left(A_{r}\right)=X_{r} \cap\left\{\phi \in \mathcal{W}^{2, r}(\Omega) ; \phi=0 \text { on } \partial \Omega\right\},
$$

generates on $X_{r}$ an analytic semigroup $\left\{e^{-t A_{r}}\right\}$ for arbitrary $1<r<\infty$.

A complete description of the domains of fractional powers of the Stokes operator $A_{r}=-v P_{r} \Delta, D\left(A_{r}\right)=D(-\Delta) \cap X_{r}$, can be found in [20, p. 269], or in [18]. Note further, that the domains of negative powers of the operator $A_{r}$ are introduced through the relation (e.g. [20, p. 269]):

$$
D\left(A_{r}^{\beta}\right)=D\left(A_{r^{\prime}}^{-\beta}\right)^{*}, \frac{1}{r}+\frac{1}{r^{\prime}}=1 .
$$

Thus, $D\left(A_{r}^{\beta}\right), \beta<0$, is the completion of $X_{r}$ under the norm $\left\|A_{r}^{\beta} \cdot\right\|_{0, r^{\prime}}$.

It is also easy to see, that the resolvent of the operator $A_{2}$ fulfills an estimate: $\Re\left(\sigma\left(A_{2}\right)\right) \geq v \lambda_{1}$, where $\lambda_{1}$ is the first positive eigenvalue of $-\Delta$ in $L^{2}(\Omega)$ considered with the Dirichlet boundary condition. The same estimate remains valid for the operator considered in $L^{r}(\Omega)$ with any $r \in(1, \infty)$. It follows further from [20, Lem. 3.1], that the resolvent of $A_{r}$ is compact, also the embeddings $D\left(A_{r}^{\beta}\right) \subset D\left(A_{r}^{\alpha}\right)$ are compact when $0<\alpha<\beta$ ([21, Th. 1.4.8]). In fact the operator $A=A_{2}$ (we skip the subscript further for simplicity) is self-adjoint in the Hilbert space $\mathcal{L}^{2}(\Omega)$; see e.g. [17,20]. For such type operators the powers of the order $(1+\alpha)$ have similar properties; in particular they are also sectorial operators.

Consequently, the operators $A_{r}, 1<r<\infty$, are sectorial positive. Fractional powers of the order $1+\alpha$ for such operators are introduced through the Balakrishnan formula $([27,34])$ :

$$
A^{1+\alpha} \phi=\frac{2 \sin (\pi \alpha)}{\alpha \pi} \int_{0}^{\infty} \lambda^{\alpha}\left[A(\lambda+A)^{-1}\right]^{2} \phi d \lambda, \phi \in D\left(A^{2}\right),
$$


We recall next the, specific for the $\mathrm{N}-\mathrm{S}$ equation, standard a priori estimate. Calculating on regular solutions (which extends to weak solutions), it is obtained multiplying (1.1) in $\left[L^{2}(\Omega)\right]^{3}$ through $u$, to get:

$$
\frac{1}{2} \frac{d}{d t}\|u\|_{\mathcal{L}^{2}(\Omega)}^{2}=-v\|\nabla u\|_{\mathcal{L}^{2}(\Omega)}^{2}-\int_{\Omega} \nabla p \cdot u d x+\int_{\Omega} f \cdot u d x,
$$

since the nonlinear component vanish in that calculation due to condition $\operatorname{div} u=0$ :

$$
\sum_{j=1}^{3} \int_{\Omega} \sum_{i=1}^{3} u_{i} \frac{\partial u_{j}}{\partial x_{i}} u_{j} d x=-\frac{1}{2} \int_{\Omega} \sum_{i=1}^{3} \frac{\partial u_{i}}{\partial x_{i}} \sum_{j=1}^{3} u_{j}^{2} d x=0 .
$$

The term $\int_{\Omega} \nabla p \cdot u d x$ is transformed as follows:

$$
\begin{aligned}
\int_{\Omega} \nabla p \cdot u d x & =\int_{\Omega}\left(\frac{\partial p}{\partial x_{1}} u_{1}+\frac{\partial p}{\partial x_{2}} u_{2}+\frac{\partial p}{\partial x_{3}} u_{3}\right) d x \\
& =-\int_{\Omega} p \text { divudx }=0 .
\end{aligned}
$$

Consequently, for divergence-free $f$, an $\mathcal{L}^{2}(\Omega)$ estimate is obtained:

$$
\begin{aligned}
\frac{1}{2} \frac{d}{d t}\|u\|_{\mathcal{L}^{2}(\Omega)}^{2} & =-v\|\nabla u\|_{\mathcal{L}^{2}(\Omega)}^{2}+\int_{\Omega} f \cdot u d x \leq-\frac{v}{2}\|\nabla u\|_{\mathcal{L}^{2}(\Omega)}^{2}+\frac{c_{P}}{2 v}\|f\|_{\mathcal{L}^{2}(\Omega)}^{2} \\
& \leq-\frac{v}{2 c_{P}}\|u\|_{\mathcal{L}^{2}(\Omega)}^{2}+\frac{c_{P}}{2 v}\|f\|_{\mathcal{L}^{2}(\Omega)}^{2},
\end{aligned}
$$

thanks to the Poincaré inequality. Integrating the above, we obtain:

$$
\|u(t)\|_{\mathcal{L}^{2}(\Omega)}^{2} \leq\left\|u_{0}\right\|_{\mathcal{L}^{2}(\Omega)}^{2} e^{-\frac{v t}{c_{P}}}+\left(\frac{c_{P}}{v}\right)^{2}\|f\|_{\mathcal{L}^{2}(\Omega)}^{2}\left(1-e^{-\frac{v t}{c_{P}}}\right)
$$

where $c_{P}$ denotes the constant in the Poincare inequality. Having already the last estimate one can return to (1.10), to see that

$$
v\|u\|_{L^{2}\left(0, T ;\left[H_{0}^{1}(\Omega)\right]^{3}\right)}^{2} \leq \frac{c_{P}}{v} T\|f\|_{\mathcal{L}^{2}(\Omega)}^{2}+\left\|u_{0}\right\|_{\mathcal{L}^{2}(\Omega)}^{2}
$$

for arbitrary $T>0$. These are the strongest natural a priori estimates known for solutions of the N-S equation.

Remark 1.2 Note that similar estimates are also valid for solutions of the fractal approximations (1.2), with only one difference that the $\left[H_{0}^{1}(\Omega)\right]^{3}$ norm is replaced with the $\left[H_{0}^{1+\alpha}(\Omega)\right]^{3}$ norm. Consequently, the estimates (1.11) and (1.12) are valid for all the solutions $u^{\alpha}$ uniformly in $\alpha>0$. 


\subsection{Local in Time Solvability of the 3-D and 2-D N-S Problems}

We will rewrite $\mathrm{N}-\mathrm{S}$ equation in a form of an abstract parabolic equation with sectorial positive operator and solve it using Dan Henry's approach ([3,21]). I want to make a comment here, that using such approach we have fairly large choice of phase spaces (= space where solution varies). In fact, calling here paper [35], as far as we consider the corresponding to (1.3) linear Cauchy problem with sectorial positive operator:

$$
\begin{aligned}
u_{t}+A u & =0, t>0, \\
u(0) & =u_{0},
\end{aligned}
$$

we can 'set it' at any level of the fractional power scale $X^{\beta}=D\left((-A)^{\beta}\right), \beta \in \mathbb{R}$, corresponding to $-A$ (see $[1$, Section V.2] for an extension of that idea). When we move to the semilinear problem (1.3) with nonlinearity $F$ subordinated to $A$, it is an art to choose the proper level at that scale to be the phase space for semilinear problem. For that, we need to consider a priori estimates available for the specific equation, usually of physical origin, e.g. following from energy decay or conservation of mass valid in the process described through the equation. The full semilinear problem will be next written abstractly as

$$
\begin{aligned}
u_{t}+A u & =F(u), t>0, \\
u(0) & =u_{0} .
\end{aligned}
$$

The standard way to set the problem (1.1) in the above setting, in $L^{2}(\Omega)$ (see e.g. $[17,20,37])$, is to apply to the equation the projector $P=P_{2}:\left[L^{2}(\Omega)\right]^{3} \rightarrow H$, where $H$ is the closure in $\left[L^{2}(\Omega)\right]^{3}$ of the set of divergence-free functions $\{u \in$ $\left[C_{0}^{\infty}(\Omega)\right]^{3} ;$ divu $\left.=0\right\}$. The pressure term disappears then from the equation. The realization $A$ of the Stokes operator acts from $D(A) \rightarrow H$. We also introduce the energy space $V=\left\{u \in\left[H_{0}^{1}(\Omega)\right]^{3} ;\right.$ divu $\left.=0\right\}$, and the simplified notation for the nonlinearity: $F(u)=-P(u \cdot \nabla) u$.

Operator $A=A_{2}$ has an associated scale of fractional order spaces $X^{\beta} \subset$ $\left[H^{2 \beta}(\Omega)\right]^{3}, \beta \geq 0$. The realizations of $A$ in $X^{\beta}$ act from $D\left(A^{\beta}\right)=X^{\beta+1} \rightarrow X^{\beta}$ and are sectorial positive operators (see e.g. [17,21,34,39]).

We will rewrite the classical N-S equation in an equivalent form, using the property of the divergence-free functions. We have:

$$
\begin{aligned}
& u_{j t}=v \Delta u_{j}-\frac{\partial p}{\partial x_{j}}-\sum_{i=1}^{3} \frac{\partial\left(u_{i} u_{j}\right)}{\partial x_{i}}+f_{j}, \quad \text { divu }=0, \quad x \in \Omega, t>0, \quad j=1,2,3, \\
& \begin{array}{l}
u=0, \quad t>0, \quad x \in \partial \Omega \\
u(0, x)=u_{0}(x),
\end{array}
\end{aligned}
$$

where $u=\left(u_{1}, u_{2}, u_{3}\right)$.

We need to emphasize here, that all the technical tools needed for local in time solvability of the N-S equation (in standard spaces), like sectoriality of the Stokes 
operator, description of its domain and needed estimates, were reported in [17, 18,20]. We recall next an estimate, important for further calculations, borrowed from [20, Lemma 2.1]. A similar observation was given also in [21, p. 18] in dimension one, see also the review of earlier results in [20, p. 271].

Corollary 1.3 For each $j, 1 \leq j \leq N$, the operator $A^{-\frac{1}{2}} P \frac{\partial}{\partial x_{j}}$ extends uniquely to a bounded linear operator from $\left[L^{r}(\Omega)\right]^{N}$ to $X_{r}, 1<r<\infty$. Consequently, the following estimate holds:

$$
\left\|A^{-\frac{1}{2}} P(u \cdot \nabla) v\right\|_{\left[L^{r}(\Omega)\right]^{N}} \leq M(r)\left\|\left|u\|v \mid\|_{\left[L^{r}(\Omega)\right]^{N}} .\right.\right.
$$

Observation 1.4 We have also the following estimates used further in the text. From [20, Lemma 2.1] we get, for all $N \in \mathbb{N}$ :

$$
\begin{aligned}
\left\|A^{-\frac{1}{2}} P(u \cdot \nabla) v\right\|_{\left[L^{2}(\Omega)\right]^{N}} & \leq c\left\|\left|u\|v \mid\|_{\left[L^{2}(\Omega)\right]^{N}} \leq c\|u\|_{\left[L^{4}(\Omega)\right]^{N}}\|v\|_{\left[L^{4}(\Omega)\right]^{N}},\right.\right. \\
\|P(u \cdot \nabla) v\|_{\left[L^{2}(\Omega)\right]^{N}} & \leq c\|u\|_{\left[L^{4}(\Omega)\right]^{N}}\|\nabla v\|_{\left[L^{4}(\Omega)\right]^{N}} .
\end{aligned}
$$

Now, for any $\delta \in\left(0, \frac{1}{2}\right)$, using the theory of interpolation;

$$
\begin{aligned}
\left\|A^{-\delta} P(u \cdot \nabla) v\right\|_{\left[L^{2}(\Omega)\right]^{N}} & \leq c\left\|A^{-\frac{1}{2}} P(u \cdot \nabla) v\right\|_{\left[L^{2}(\Omega)\right]^{N}}^{2 \delta}\|P(u \cdot \nabla) v\|_{\left[L^{2}(\Omega)\right]^{N}}^{1-2 \delta} \\
& \leq c\|u\|_{\left[L^{4}(\Omega)\right]^{N}}^{1+2 \delta}\|\nabla v\|_{\left[L^{4}(\Omega)\right]^{N}}^{1-2 \delta} .
\end{aligned}
$$

In a similar way, starting from the estimates:

$$
\begin{aligned}
& \left\|A^{-\frac{1}{2}} P(u \cdot \nabla) v\right\|_{\left[L^{2}(\Omega)\right]^{N}} \leq c\left\|\left|u\|v \mid\|_{\left[L^{2}(\Omega)\right]^{N}} \leq c\|u\|_{\left[L^{6}(\Omega)\right]^{N}}\|v\|_{\left[L^{3}(\Omega)\right]^{N}},\right.\right. \\
& \|P(u \cdot \nabla) v\|_{\left[L^{2}(\Omega)\right]^{N}} \leq c\|u\|_{\left[L^{6}(\Omega)\right]^{N}}\|\nabla v\|_{\left[L^{3}(\Omega)\right]^{N}},
\end{aligned}
$$

for any $\delta \in\left(0, \frac{1}{2}\right)$, we get:

$$
\begin{aligned}
\left\|A^{-\delta} P(u \cdot \nabla) v\right\|_{\left[L^{2}(\Omega)\right]^{N}} & \leq c\left\|A^{-\frac{1}{2}} P(u \cdot \nabla) v\right\|_{\left[L^{2}(\Omega)\right]^{N}}^{2 \delta}\|P(u \cdot \nabla) v\|_{\left[L^{2}(\Omega)\right]^{N}}^{1-2 \delta} \\
& \leq c\|u\|_{\left[L^{6}(\Omega)\right]^{N}}\|u\|_{\left[L^{3}(\Omega)\right]^{N}}^{2 \delta}\|\nabla v\|_{\left[L^{3}(\Omega)\right]^{N}}^{1-2 \delta}
\end{aligned}
$$

The above estimates (1.16), (1.18), are valid for all the space dimensions $N=$ $2,3,4, \ldots$ They can be extended further, using Sobolev type estimates, in a way depending on $N$.

For local in time solvability, we will set the problem (1.13) in the base space $X^{-\frac{1}{4}}$ for the space dimension $N=2$, and in the base space $X^{-\frac{1}{8}}$ for the space dimension $N=3$. The corresponding phase spaces will be; $\left.X^{\frac{1}{2}^{+}} \subset\left[H^{1^{+}}(\Omega)\right]^{2}\right)$ in case $N=2$, and $X^{3^{3}} \subset\left[H^{3^{2}}(\Omega)\right]^{3}$ ) in case $N=3$ (e.g. [20, Proposition 1.4]). Note that, in both cases, the phase spaces are contained in the space $\left[L^{\infty}(\Omega)\right]^{N}$. Note also, there 
is another possible choice of the phase spaces (e.g. [20]), if we decide to work in the spaces $\left[L^{r}(\Omega)\right]^{N}, N=2,3$, with $r>N$.

We will formulate now the corresponding local existence results for $N=2,3$.

Case $N=3$. The main tool is the estimate taken from [20, Lemma 2.2] (with $\left.\delta=\frac{1}{8}, \theta=\rho=\frac{3}{4}\right)$ :

$$
\left\|A^{-\frac{1}{8}} P(u \cdot \nabla) v\right\|_{\left[L^{2}(\Omega)\right]^{3}} \leq M\left\|A^{\frac{3^{+}}{4}} u\right\|_{\left[L^{2}(\Omega)\right]^{3}}\left\|A^{\frac{3^{+}}{}} v\right\|_{\left[L^{2}(\Omega)\right]^{3}} .
$$

Since the form above is bi-linear, we have also the following consequences of the last estimate:

$$
\begin{aligned}
& \left\|A^{-\frac{1}{8}} P((u-v) \cdot \nabla) v\right\|_{\left[L^{2}(\Omega)\right]^{3}} \leq M\left\|A^{\frac{3}{4}^{+}}(u-v)\right\|_{\left[L^{2}(\Omega)\right]^{3}}\left\|A^{\frac{3}{4}^{+}} v\right\|_{\left[L^{2}(\Omega)\right]^{3}}, \\
& \left\|A^{-\frac{1}{8}} P(u \cdot \nabla)(u-v)\right\|_{\left[L^{2}(\Omega)\right]^{3}} \leq M\left\|A^{\frac{3}{4}^{+}} u\right\|_{\left[L^{2}(\Omega)\right]^{3}}\left\|A^{\frac{3}{4}^{+}}(u-v)\right\|_{\left[L^{2}(\Omega)\right]^{3}} .
\end{aligned}
$$

Consequently, the nonlinear term $F(u)+P f=-P(u \cdot \nabla) u+P f$ acts from $D\left(A^{\frac{3}{4}^{+}}\right) \subset\left[H^{\frac{3^{2}}{}}(\Omega)\right]^{3}$ into $D\left(A^{-\frac{1}{8}}\right)$ as a map, Lipschitz continuous on bounded subsets of $D\left(A^{\frac{3}{4}^{+}}\right)$. Indeed, for $u, v$ varying in a bounded set $B \subset D\left(A^{\frac{3}{4}^{+}}\right)$,

$$
\begin{aligned}
& \left\|A^{-\frac{1}{8}} F(u-v)\right\|_{\left[L^{2}(\Omega)\right]^{3}} \\
& \leq M\left\|A^{\frac{3}{4}^{+}}(u-v)\right\|_{\left[L^{2}(\Omega)\right]^{3}}\left(\left\|A^{3^{\frac{3}{4}}} u\right\|_{\left[L^{2}(\Omega)\right]^{3}}+\left\|A^{3^{+}} v\right\|_{\left[L^{2}(\Omega)\right]^{3}}\right) \\
& \leq C(B)\left\|A^{\frac{3}{4}^{+}}(u-v)\right\|_{\left[L^{2}(\Omega)\right]^{3}} .
\end{aligned}
$$

According to abstract theory in $[3,21]$ and thanks to the properties of the Stokes operator as reported in $[17,20]$, this suffices to obtain a local in time solution of the 3-D equation (1.1) (also of 3-D equation (2.6)), more precisely we have:

Theorem 1.5 When Pf $\in D\left(A^{-\frac{1}{8}}\right), u_{0} \in D\left(A^{3^{\frac{3}{4}}}\right)$, then there exists a unique local in time mild solution $u(t)$ to $(1.1)$ in the phase space $D\left({A^{\frac{3^{4}}{}}}^{+}\right) \subset\left[{H^{\frac{3}{2}}}^{+}(\Omega)\right]^{3}$. Moreover,

$u \in C\left([0, \tau) ; D\left({A^{\frac{3}{4}}}^{+}\right)\right) \cap C\left((0, \tau) ; D\left(A^{\frac{7}{8}}\right)\right), u_{t} \in C\left((0, \tau) ; D\left(A^{7^{-}}\right)\right)($

Here $\tau>0$ is the 'life time' of that local in time solution. Moreover, the Cauchy formula is satisfied:

$$
u(t)=e^{-A t} u_{0}+\int_{0}^{t} e^{-A(t-s)} F(u(s)) d s, \quad t \in[0, \tau),
$$

where $e^{-A t}$ denotes the linear semigroup corresponding to the operator A. We need also to mention that the considered here mild solutions have additional regularity 
properties, as described in particular in [3, p. 218]; here $u \in C^{1}\left((0, \tau) ; D\left(A^{7^{-}}\right)\right)$. This property is used in the calculations below.

Case $N=2$. We will use a version of the estimate in [20, Lemma 2.2] (with $\left.\delta=\frac{1}{4}, \theta=\rho=\frac{1}{2}\right)$ :

$$
\left\|A^{-\frac{1}{4}} P(u \cdot \nabla) v\right\|_{\left[L^{2}(\Omega)\right]^{2}} \leq M\left\|A^{\frac{1}{2}^{+}} u\right\|_{\left[L^{2}(\Omega)\right]^{3}}\left\|A^{\frac{1}{2}^{+}} v\right\|_{\left[L^{2}(\Omega)\right]^{2}} .
$$

Since the form above is bi-linear, we have also the following consequences of the last estimate:

$$
\begin{aligned}
& \left\|A^{-\frac{1}{4}} P((u-v) \cdot \nabla) v\right\|_{\left[L^{2}(\Omega)\right]^{2}} \leq M\left\|A^{\frac{1}{2}^{+}}(u-v)\right\|_{\left[L^{2}(\Omega)\right]^{2}}\left\|A^{\frac{1}{2}^{+}} v\right\|_{\left[L^{2}(\Omega)\right]^{2}}, \\
& \left\|A^{-\frac{1}{4}} P(u \cdot \nabla)(u-v)\right\|_{\left[L^{2}(\Omega)\right]^{2}} \leq M\left\|A^{\frac{1}{2}^{+}} u\right\|_{\left[L^{2}(\Omega)\right]^{2}}\left\|A^{\frac{1}{2}^{+}}(u-v)\right\|_{\left[L^{2}(\Omega)\right]^{2}} .
\end{aligned}
$$

Consequently, the nonlinear term $F(u)+P f=-P(u \cdot \nabla) u+P f$ acts from $D\left(A^{\frac{1}{2}}{ }^{+}\right) \subset\left[H^{1^{+}}(\Omega)\right]^{2}$ into $D\left(A^{-\frac{1}{4}}\right)$ as a map, Lipschitz continuous on bounded subsets of $D\left(A^{\frac{1}{2}^{+}}\right)$. According to [3,21], this suffices to obtain a local in time solution of the 2-D equation (1.1) (also of 2-D equation (1.2)), more precisely:

Theorem 1.6 When Pf $\in D\left(A^{-\frac{1}{4}}\right), u_{0} \in D\left(A^{1^{2}}\right) \subset\left[H^{1^{+}}(\Omega)\right]^{2}$, then there exists a unique local in time mild solution $u(t)$ to $(1.1)$ in the phase space $D\left(A^{1^{2}}\right) \subset$ $\left[H^{1^{+}}(\Omega)\right]^{2}$. Moreover,

$u \in C\left([0, \tau) ; D\left(A^{\frac{1}{2}^{+}}\right)\right) \cap C\left((0, \tau) ; D\left(A^{\frac{3}{4}}\right)\right), u_{t} \in C\left((0, \tau) ; D\left(A^{\frac{3^{-}}{4}}\right)\right)$.

Here $\tau>0$ is the 'life time' of that local in time solution. Moreover, the Cauchy formula is satisfied:

$$
u(t)=e^{-A t} u_{0}+\int_{0}^{t} e^{-A(t-s)} F(u(s)) d s, \quad t \in[0, \tau),
$$

where $e^{-A t}$ denotes the linear semigroup corresponding to the operator A.

Remark 1.7 Another choices of the pairs base/phase spaces are possible [20]. In particular, setting for $N=2:\left(\delta=1-v, \theta=\rho=\frac{v}{2}, v=0^{+}\right)$in [20, Lemma 2.2], one has that $F: D\left(A^{\frac{v}{2}}\right) \rightarrow D\left(A^{-1+v}\right)$. For $N=3$, setting there: $\left(\delta=\frac{1}{2}, \theta=\rho=\frac{3}{8}\right)$, one has that $F: D\left(A^{\frac{3}{8}}\right) \rightarrow D\left(A^{-\frac{1}{2}}\right)$, and $F$ is Lipschitz continuous on bounded sets. Corresponding local solvability results follow immediately. 


\subsection{Properties of 2-D and 3-D N-S Equation with Respect to the Known a Priori Bound}

We will consider now criticality of the N-S equation (1.1) in the sense stated e.g. in $[3,40,41]$, that means 'the nonlinear term is of the same order as the main part operator with respect to the given a priori estimate'. To exhibit this concept better we will assume in the definition below that (1.3) is stated in a Banach 'base' space denoted $Z, A$ is a sectorial positive operator in $Z$ and $F$ is a map Lipschitz continuous on bounded sets from $Z^{\beta}$ into $Z$ where $\beta \in[0,1)$. In particular we will consider in this setting $Z^{\beta}$ solutions as in [3, p. 55].

Definition 1.8 The problem (1.3) will be called sub-critical with respect to the given a priori estimate in $Y$, if for all its possible $Z^{\beta}$ solutions an estimate holds

$$
\exists_{\theta \in([0,1]} \exists_{\text {nondecreasing } g:[0, \infty) \rightarrow[0, \infty)}\|F(u(t))\|_{Z} \leq g\left(\|u(t)\|_{Y}\right)\left(1+\left\|A^{\beta} u(t)\right\|_{Z}^{\theta}\right),
$$

for all $t \in\left(0, \tau_{u_{0}}\right)$, the life time of the considered $Z^{\beta}$ solution.

The problem (1.3) will be called critical with respect to the given a priori estimate in $Y$ if, instead of (1.25), for all its possible $Z^{\beta}$ solutions an estimate holds

$$
\exists_{\text {nondecreasing } g:[0, \infty) \rightarrow[0, \infty)}\|F(u(t))\|_{Z} \leq g\left(\|u(t)\|_{Y}\right)\left(1+\left\|A^{1} u(t)\right\|_{Z}\right),
$$

for all $t \in\left(0, \tau_{u_{0}}\right)$, the life time of the considered $Z^{\beta}$ solution.

Using the abstract setting as in Definition 1.8 we now indicate that the 3-D N-S can manifest a different behavior; namely,

$$
\exists_{\Theta>1} \exists_{\text {nondecreasing } g:[0, \infty) \rightarrow[0, \infty)} \quad\|F(u(t))\|_{Z} \leq g\left(\|u(t)\|_{Y}\right)\left(1+\left\|A^{1} u(t)\right\|_{Z}^{\Theta}\right),
$$

for all $t \in\left(0, \tau_{u_{0}}\right)$.

Case $N=3$. In case of the Navier-Stokes equation (1.1) the mentioned above a priori estimate will be the $Y=\left[L^{2}(\Omega)\right]^{N}$ estimate (1.11).

Following first (1.19), thus choosing $X^{-\frac{1}{8}}$ and $X^{\frac{3^{4}}{4}}$ to play a role of $Z$ and $Z^{\beta}$ respectively, we will see that (1.27) holds for (1.1) with respect to the standard $L^{2}$ estimate. Indeed, the estimate (1.18) written for the local solution $u=u(t)$ obtained in Theorem 1.5 extends for $N=3$, with the use of the Nirenberg-Gagliardo type estimates, to:

$$
\begin{aligned}
\left\|A^{-\frac{1}{8}} P(u \cdot \nabla) u\right\|_{\left[L^{2}(\Omega)\right]^{3}} & \leq c\|u\|_{\left[L^{6}(\Omega)\right]^{3}}\|u\|_{\left[L^{3}(\Omega)\right]^{3}}^{\frac{1}{4}}\|\nabla u\|_{\left[L^{3}(\Omega)\right]^{3}}^{\frac{3}{4}} \\
& \leq c\|u\|_{\left[H^{\frac{7}{4}}(\Omega)\right]^{3}}^{\frac{9}{7}}\|u\|_{\left[L^{2}(\Omega)\right]^{3}}^{\frac{5}{7}},
\end{aligned}
$$

which gives the exponent $\Theta$ above equal $\frac{9}{7}$. 
On the other hand, using the estimate of Corollary 1.3, we will find for the 3-D N-S equation that

$$
\begin{aligned}
\|P(u \cdot \nabla) u\|_{X^{-\frac{1}{2}}} & =\left\|A^{-\frac{1}{2}} P(u \cdot \nabla) u\right\|_{\left[L^{2}(\Omega)\right]^{3}} \leq M\left\||u|^{2}\right\|_{\left[L^{2}(\Omega)\right]^{3}} \leq c\|u\|_{\left[L^{4}(\Omega)\right]^{3}}^{2} \\
& \leq c\|u\|_{\left[H^{1}(\Omega)\right]^{3}}^{\frac{3}{2}}\|u\|_{\left[L^{2}(\Omega)\right]^{3}}^{\frac{1}{2}}
\end{aligned}
$$

in which case $\Theta=\frac{3}{2}$.

Finally, for the further use, we will check how large the exponent $s>1$ should be, for the 'strengthen diffusion' of the form $A^{s}$ replacing the usual operator $A$ in (1.1) to make the $L^{2}(\Omega)$ estimate critical. Using again Corollary 1.3 we get that

$$
\left\|A^{-\frac{1}{2}} P(u \cdot \nabla) u\right\|_{\left[L^{2}(\Omega)\right]^{3}} \leq M\left\||u|^{2}\right\|_{\left[L^{2}(\Omega)\right]^{3}} \leq c\|u\|_{\left[H^{2 s-1}(\Omega)\right]^{3}}^{\frac{3}{2(2 s-1)}}\|u\|_{\left[L^{2}(\Omega)\right]^{3}}^{\frac{4 s-5}{4 s-2}} .
$$

We find such critical value of $s$ from the condition; $\frac{3}{2(2 s-1)}=1$. Consequently, $s=\frac{5}{4}$.

Remark 1.9 Consideration as in the above case indicates (although it does not prejudge) that the 3-D N-S may behave in a supercritical manner with respect to $L^{2}$ estimate of the solutions, which in turn leads to an open question if there exists a mechanism in the 3-D N-S equation that prevents the development of a singularity (see also Remark 3.7).

Case $N=2$. Using Corollary 1.3 it is easy to see criticality of the 2-D NavierStokes equation. More precisely, to verify that its nonlinearity is critical with respect to the standard $L^{2}(\Omega)$ a priori estimates, as a map from $X^{\frac{1}{2}} \subset\left[H^{1}(\Omega)\right]^{2}$ to $X^{-\frac{1}{2}}$. Indeed,

$$
\begin{aligned}
\|P(u \cdot \nabla) u\|_{X^{-\frac{1}{2}}} & \leq\left\|A^{-\frac{1}{2}} P(u \cdot \nabla) u\right\|_{\left[L^{2}(\Omega)\right]^{2}} \leq M\left\||u|^{2}\right\|_{\left[L^{2}(\Omega)\right]^{2}} \\
& \leq c\|u\|_{\left[L^{4}(\Omega)\right]^{2}}^{2} \leq\|u\|_{\left[H^{1}(\Omega)\right]^{2}}\|u\|_{\left[L^{2}(\Omega)\right]^{2}}=c\left(\|u\|_{\left.\left[L^{2}(\Omega)\right]^{2}\right)\|u\|_{X^{\frac{1}{2}}}^{1}},\right.
\end{aligned}
$$

and no better estimate (with exponent smaller than 1) seems possible.

Observation 1.10 Using the estimate (1.16) with $\delta=\frac{1}{4}$, we will show that the nonlinearity in 2-D N-S equation is critical as a map from $X^{\frac{3}{4}} \subset\left[H^{\frac{3}{2}}(\Omega)\right]^{2}$ to $X^{-\frac{1}{4}}$. Indeed, from (1.16) with $\delta=\frac{1}{4}, N=2$,

$$
\left\|A^{-\frac{1}{4}} P(u \cdot \nabla) u\right\|_{\left[L^{2}(\Omega)\right]^{2}} \leq c\|u\|_{\left[L^{4}(\Omega)\right]^{2}}^{\frac{3}{2}}\|\nabla u\|_{\left[L^{4}(\Omega)\right]^{2}}^{\frac{1}{2}} \leq c\|u\|_{\left[L^{2}(\Omega)\right]^{2}}\|u\|_{\left[H^{\frac{3}{2}}(\Omega)\right]^{2}},
$$


where the Nirenberg-Gagliardo type estimates were used:

$$
\begin{aligned}
\|\phi\|_{L^{4}(\Omega)} & \leq c\|\phi\|_{H^{\frac{3}{2}(\Omega)}}^{\frac{1}{3}}\|\phi\|_{L^{2}(\Omega)}^{\frac{2}{3}}, \\
\|\phi\|_{W^{1,4}(\Omega)} & \leq c\|\phi\|_{H^{\frac{3}{2}(\Omega)}} .
\end{aligned}
$$

No better estimates seems possible.

Note further, that in 2-D the nonlinearity is also critical with respect to the $L^{2}(\Omega)$ a priori estimate as a map between $X^{1}=D(A) \subset\left[H^{2}(\Omega)\right]^{2}$ and $X$. Indeed, the following estimate holds,

$$
\|P(u \cdot \nabla) u\|_{X} \leq c\|u\|_{\left[L^{4}(\Omega)\right]^{2}}\|\nabla u\|_{\left[L^{4}(\Omega)\right]^{2}} \leq c\|u\|_{\left[H^{2}(\Omega)\right]^{2}}\|u\|_{\left[L^{2}(\Omega)\right]^{2}},
$$

and no smaller exponent on the $H^{2}(\Omega)$ norm seems possible.

\section{Global in Time Solutions in 3-D: Small Data}

As well known (e.g. [41], [3, Chapters 3,5]), global in time extendibility of the local mild solution constructed in the Theorem 1.5 is possible provided we have sufficiently well a priori estimates that prevents the $D\left({A^{\frac{3}{4}}}^{+}\right) \subset\left[H^{\frac{3}{2}^{+}}(\Omega)\right]^{3}$ norm of the solution to blow up in a finite time. We will show next such type estimate, in $\left[H^{1}(\Omega)\right]^{3}$, for solutions of the 3-D N-S equation when the data: $u_{0}$ and $f$ are smooth and sufficiently small; see e.g. [33, Theorem 6.8, Chapter 1] for such result. The estimate will be used later to construct global in time solutions of the N-S with small data. Another approach to that problem in arbitrary dimension $N$, using estimates on integral equation, was presented in [2].

Theorem 2.1 If $u_{0} \in D(A) \subset\left[H^{2}(\Omega)\right]^{3}$ and $f \in\left[L^{2}(\Omega)\right]^{3}$ fulfill the 'smallness restriction' (2.4), then the $\left[H^{1}(\Omega)\right]^{3}$ norms of the solutions $u$ are bounded uniformly in time $t \geq 0$.

Proof It is known (e.g. [3,21]), that the local in time solutions $u$ are regularized for $t>0$ (through the equation) into the space $D(A) \subset\left[H^{2}(\Omega)\right]^{3}$. When the initial data $u_{0} \in D(A)$, they simply vary in $D(A)$ for $t \geq 0$ small, until possible blow-up time $t\left(\alpha, u_{0}\right)$. We want to show that, to small $u_{0}$ and $f$ correspond small solutions, in the $\left[H^{1}(\Omega)\right]^{3}$ norm (uniformly in time).

To get estimate of the solution $u$ in $\left[H^{1}(\Omega)\right]^{3}$ multiply (1.2) by $A u$ to obtain:

$$
\begin{aligned}
<u_{t}, A u>_{\left[L^{2}(\Omega)\right]^{3}}= & -<A u, A u>_{\left[L^{2}(\Omega)\right]^{3}}-<P(u \cdot \nabla) u, A u>_{\left[L^{2}(\Omega)\right]^{3}} \\
& +<P f, A u>_{\left[L^{2}(\Omega)\right]^{3}},
\end{aligned}
$$


since the pressure term vanishes. Thanks to (1.15), this gives,

$$
\begin{aligned}
\frac{1}{2} \frac{d}{d t}\|u\|_{\left[H^{1}(\Omega)\right]^{3} \leq}^{2} & -c v\|u\|_{\left[H^{2}(\Omega)\right]^{3}}^{2}+c\|u\|_{\left[W^{1,4}(\Omega)\right]^{3}}\|u\|_{\left[L^{4}(\Omega)\right]^{3}}\|u\|_{\left[H^{2}(\Omega)\right]^{3}} \\
& +\|P f\|_{\left[L^{2}(\Omega)\right]^{3}}\|u\|_{\left[H^{2}(\Omega)\right]^{3}} \\
\leq & -c v\|u\|_{\left[H^{2}(\Omega)\right]^{3}}^{2}+c_{1}\|u\|_{\left[H^{2}(\Omega)\right]^{3}}^{\frac{5}{4}}\|u\|_{\left[H^{1}(\Omega)\right]^{3}}^{\frac{7}{4}} \\
& +\|P f\|_{\left[L^{2}(\Omega)\right]^{3}}\|u\|_{\left[H^{2}(\Omega)\right]^{3}} \\
\leq & -\frac{c^{\prime} v}{2}\|u\|_{\left[H^{1}(\Omega)\right]^{3}}^{2}+C_{v}\left(\|u\|_{\left[H^{1}(\Omega)\right]^{3}}^{\frac{14}{3}}+\|P f\|_{\left[L^{2}(\Omega)\right]^{3}}^{2}\right),
\end{aligned}
$$

with the standard use of Young's inequality, and the embedding $H^{2}(\Omega) \subset H^{1}(\Omega)$ (constant $c^{\prime}$ ). Denoting; $y(t):=\|u(t)\|_{\left[H^{1}(\Omega)\right]^{3}}^{2}$, we arrive at the differential inequality (e.g. [42]):

$$
\begin{aligned}
\frac{1}{2} y^{\prime}(t) & \leq-\frac{c^{\prime} v}{2} y(t)+C_{v} y^{\frac{7}{3}}(t)+C_{v}\|P f\|_{\left[L^{2}(\Omega)\right]^{3}}^{2}, \\
y(0) & =\|u(0)\|_{\left[H^{1}(\Omega)\right]^{3}}^{2} .
\end{aligned}
$$

Analyzing its right hand side, real function $g(z)=-\frac{c^{\prime} v}{2} z+C_{\nu} z^{\frac{7}{3}}+C_{v}\|P f\|_{\left[L^{2}(\Omega)\right]^{3}}^{2}$, we see that $g(0)=C_{v}\|P f\|_{\left[L^{2}(\Omega)\right]^{3}}^{2}>0, g^{\prime}(0)<0$, and $g$ has a minimum for the argument $z_{\min }=\left(\frac{3 c^{\prime} v}{14 C_{v}}\right)^{\frac{3}{4}}$, with $g\left(z_{\min }\right)<0$ when the 'free term' $C_{v}\|P f\|_{\left[L^{2}(\Omega)\right]^{3}}^{2}$ is small. More precisely, to keep the value of $y(t)$ bounded for all positive times, we need to assume the smallness hypothesis: Let the data: $\|u(0)\|_{\left[H^{1}(\Omega)\right]^{3}}^{2}$ and $\|P f\|_{\left[L^{2}(\Omega)\right]^{3}}^{2}$ be so small, that:

$$
\begin{aligned}
& g\left(z_{\text {min }}\right)<0, \text { equivalently }\|P f\|_{\left[L^{2}(\Omega)\right]^{3}}^{2}<\frac{4}{3} z_{\text {min }}^{\frac{7}{3}}, \text { and } \\
& \left\|u_{0}\right\|_{\left[H^{1}(\Omega)\right]^{3}}^{2} \leq z_{\min }=\left(\frac{3 c^{\prime} v}{14 C_{v}}\right)^{\frac{3}{4}} .
\end{aligned}
$$

Note that $\left(\frac{v}{C_{v}}\right)^{\frac{3}{4}}$ is proportional to $v^{2}$. Consequently we obtain the bound

$$
\|u(t)\|_{\left[H^{1}(\Omega)\right]^{3}}^{2} \leq z_{\min } \text { valid for all } t \geq 0 .
$$

With the last assumption, the smooth local solutions $u(t)$, introduced in Theorem 3.1 are bounded in $\left[H^{1}(\Omega)\right]^{3}$ uniformly in $t \geq 0$. Note, that a bit more accurate bounds in (2.4) are possible if one compare the data with the two positive zeros of the function $g$. See also the corresponding restrictions formulated in [37, Theorem 3.7]. 


\subsection{Regularization of the 3-D N-S Equation}

It was shown above that in the 3-D case the N-S equation (1.1) may behave with respect to the standard estimate according to (1.27). Global in time extendibility of the local solutions constructed in Theorem 1.5 is in general unknown (unless for small data). The viscosity term in the equation (1.1) together with the standard a priori estimate are not strong enough to control the nonlinearity. In the present subsection we discuss an approximation/regularization (with stronger diffusion term) of the 3-D N-S equation, for which the $L^{2}(\Omega)$ estimates (same as for the original equation) are sufficient to make such problems sub-critical. The idea of such regularization was proposed first by Lions in [33, Chapter 1, Remarque 6.11].

We consider approximation of the original 3-D Navier-Stokes equation having global in time, unique and regular solutions. Consider namely the approximation/regularization of (1.1) having the form:

$$
\begin{aligned}
u_{t} & =-\left(A+\epsilon A^{s}\right) u-P(u \cdot \nabla) u+P f, \quad t>0, \\
u(0, x) & =u_{0}(x),
\end{aligned}
$$

with a parameter $s>1$ (to be chosen), and $\epsilon>0$. For fixed (for a moment) parameter $\epsilon$, denote the solution to the above problem as $u^{s}$. We mean here the solution on the base space $D\left(A^{-\frac{1}{4}}\right)$, obtained in a similar way as for the original N-S equation (1.1) in Sect. 1.3.

It is clear how to determine the proper, sufficiently large, value of the exponent $s>1$ to guarantee, together with the $L^{2}(\Omega)$ estimates valid also for solutions of (2.6), that the nonlinear term is subordinated to the main part operator $-\left(A+\epsilon A^{s}\right)$. We need to compare the bound obtained for the nonlinearity with the income from the improved viscosity term. Estimate of the nonlinearity, obtained from (1.16), reads

$$
\left\|A^{-\frac{1}{4}} F\left(u^{s}(t)\right)\right\|_{\left[L^{2}(\Omega)\right]^{3}} \leq c\left\|u^{s}(t)\right\|_{\left[L^{4}(\Omega)\right]^{3}}^{\frac{3}{2}}\left\|u^{s}(t)\right\|_{\left[W^{1,4}(\Omega)\right]^{3}}^{\frac{1}{2}} \cdot
$$

Let $s>\frac{5}{4}$. With the use of the Nirenberg-Gagliardo type estimate we obtain the subordination condition

$$
\begin{aligned}
\left\|A^{-\frac{1}{4}} F\left(u^{s}(t)\right)\right\|_{\left[L^{2}(\Omega)\right]^{3}} & \leq c\left(\left\|u^{s}(t)\right\|_{\left[L^{2}(\Omega)\right]^{3}}\right)\left\|u^{s}(t)\right\|_{\left[H^{2 s-\frac{1}{2}}(\Omega)\right]^{3}}^{\theta} \\
& \leq c^{\prime}\left(\left\|u^{s}(t)\right\|_{\left[L^{2}(\Omega)\right]^{3}}\right)\left\|A^{s-\frac{1}{4}} u^{s}(t)\right\|_{\left[L^{2}(\Omega)\right]^{3}}^{\theta},
\end{aligned}
$$

where $\theta=\frac{2}{2 s-\frac{1}{2}}<1$. Consequently, the standard $L^{2}(\Omega)$ a priori estimate is sufficient to assure the global in time extendibility of such local solutions $u^{s}$ when $s>\frac{5}{4}$. We will study such approximation next.

Remark 2.2 The exponent $\frac{5}{4}$ proposed for regularization of (1.1) in [33, Chapter 1, Remarque 6.11] is the same (also, as in (1.30)). 


\subsection{3-D N-S Equation}

Let us study the introduced above regularization (2.6) of the original 3-D N-S equation with exponent $s>\frac{5}{4}$. We formulate first the corresponding existence result.

Theorem 2.3 Consider (2.6) with $s>\frac{5}{4}$ as equation in $D\left(A^{-\frac{1}{8}}\right)$. When Pf $\in$ $D\left(A^{-\frac{1}{8}}\right), u_{0} \in D\left(A^{\frac{3}{4}}{ }^{+}\right) \subset\left[H^{2^{3}}(\Omega)\right]^{3}$, then there exists a unique local in time mild solution $u^{\epsilon}(t)$ to (2.6), $s>\frac{5}{4}$, in the phase space $D\left({A^{\frac{3}{4}}}^{+}\right)$. Moreover,

$$
u^{\epsilon} \in C\left([0, \tau) ; D\left(A^{\frac{3}{4}^{+}}\right)\right) \cap C\left((0, \tau) ; D\left(A^{s-\frac{1}{4}}\right)\right), u_{t}^{\epsilon} \in C\left((0, \tau) ; D\left(A^{\left(s-\frac{1}{4}\right)^{-}}\right)\right) \text {. }
$$

Here $\tau>0$ is the 'life time' of that local in time solution. Also, the corresponding Cauchy formula is satisfied.

Furthermore, together with the standard $\left[L^{2}(\Omega)\right]^{3}$ a priori estimate valid for solutions of (2.6) uniformly in $\epsilon>0$, the obtained above local solution will be extended globally in time in the class (2.9).

The proof, similar as for Theorem 1.5, is omitted.

Passing to the limit $\epsilon \rightarrow 0^{+}$in (2.10) We will describe next shortly the process of passing to the limit, as $\epsilon \rightarrow 0^{+}$, in such approximations. The idea is similar as in $[7,8,33]$, using the parabolic regularization technique. We write the corresponding approximating equation:

$$
u_{t}=-A u-\epsilon A^{s} u+F(u)+P f
$$

with $s>\frac{5}{4}$ (but close to $\frac{5}{4}$ ), and denote its solution by $u^{\epsilon}$. Applying to it the operator $A^{-s+\frac{1}{2}}$ we get

$$
A^{-s+\frac{1}{2}} u_{t}^{\epsilon}=-A^{-s+\frac{3}{2}} u^{\epsilon}-\epsilon A^{\frac{1}{2}} u^{\epsilon}+A^{-s+\frac{1}{2}} F\left(u^{\epsilon}\right)+A^{-s+\frac{1}{2}} P f .
$$

Using to nonlinear term the estimate of [20, Lemma 2.2] with $\delta=s-\frac{1}{2}, \epsilon=s-1$, we get

$$
\begin{aligned}
\left\|A^{-\delta} F\left(u^{\epsilon}\right)\right\|_{\left[L^{2}(\Omega)\right]^{3}} & \leq c\left\|\left|u^{\epsilon}\right|^{2}\right\|_{\left[L^{z}(\Omega)\right]^{3}} \leq c^{\prime}\left\|u^{\epsilon}\right\|_{\left[L^{2 z}(\Omega)\right]^{3}}^{2} \\
& \leq c^{\prime \prime}\left\|u^{\epsilon}\right\|_{\left[H^{1}(\Omega)\right]^{3}}^{\frac{7}{2}-2 s}\left\|u^{\epsilon}\right\|_{\left[L^{2}(\Omega)\right]^{3}}^{2 s-\frac{3}{2}} \\
& =c^{\prime \prime \prime}\left\|A^{\frac{1}{2}} u^{\epsilon}\right\|_{\left[L^{2}(\Omega)\right]^{3}}^{\frac{7}{2}-2 s}\left\|u^{\epsilon}\right\|_{\left[L^{2}(\Omega)\right]^{3}}^{2 s-\frac{3}{2}},
\end{aligned}
$$

where $\frac{1}{z}=\frac{1}{2}+\frac{2 \epsilon}{3}$, consequently $z=\frac{6}{3+4(s-1)}<\frac{3}{2}$ (but close). Due to the standard $L^{2}$ estimate, all the right hand side components in $(2.11)$ belong to $L^{2}\left(0, T ;\left[L^{2}(\Omega)\right]^{3}\right)$ (in particular $-\epsilon A^{\frac{1}{2}} u$; note that $\frac{7}{2}-2 s<1$ in (2.12)). Consequently,

$$
A^{-s+\frac{1}{2}} u_{t}^{\epsilon} \in L^{2}\left(0, T ;\left[L^{2}(\Omega)\right]^{3}\right) \text {, or } u_{t}^{\epsilon} \in L^{2}\left(0, T ; D\left(A^{-s+\frac{1}{2}}\right)\right) \text {. }
$$


Thus the Lions-Aubin compactness lemma (e.g. [33, Chapter I, 5]) will be used to conclude that any sequence $\left\{u^{\epsilon_{n}}\right\}$ has a subsequence convergent almost everywhere in $(0, T) \times \Omega$, as $\epsilon_{n} \rightarrow 0^{+}$. Also, any sequence $\left\{u^{\epsilon_{n}}\right\}$ has a subsequence convergent in $L^{2}\left(0, T ;\left[H_{0}^{1^{-}}(\Omega)\right]^{3}\right)$.

Denote by $U$ the limit in $L^{2}\left(0, T ;\left[H_{0}^{1^{-}}(\Omega)\right]^{3}\right)$ of $u^{\epsilon}$, over a sequence $\epsilon_{n} \rightarrow 0^{+}$. Remember that from the standard a priori estimate written for the problem (2.6) it follows that the $D\left(A^{\frac{s}{2}}\right)$ norms of $u^{\epsilon}$ fulfill

$$
\sqrt{\epsilon}\left\|A^{\frac{s}{2}} u^{\epsilon}\right\|_{L^{2}\left(0, T ;\left[L^{2}(\Omega)\right]^{3}\right)} \leq \text { const },
$$

with const independent on $\epsilon>0$. We recall a weak formulation of the approximating equations (2.10):

$$
\begin{aligned}
<u_{t}^{\epsilon}, v>_{\left[L^{2}(\Omega)\right]^{3}=} & -<A^{\frac{1}{2}} u^{\epsilon}, A^{\frac{1}{2}} v>_{\left[L^{2}(\Omega)\right]^{3}}-\epsilon<A^{\frac{s}{2}} u^{\epsilon}, A^{\frac{s}{2}} v>_{\left[L^{2}(\Omega)\right]^{3}} \\
& +<F\left(u^{\epsilon}\right), v>_{\left[L^{2}(\Omega)\right]^{3}}+<P f, v>_{\left[L^{2}(\Omega)\right]^{3}}
\end{aligned}
$$

where $v \in D\left(A^{\frac{1}{2}}\right)$ arbitrary. Note that, due to (2.13), the second right hand side component vanishes when $\epsilon \rightarrow 0^{+}$. For the nonlinear component we have

$$
\begin{aligned}
\int_{0}^{T}<F\left(u^{\epsilon_{n}}\right), v>_{\left[L^{2}(\Omega)\right]^{3}} d t & =\int_{0}^{T}<A^{-\frac{1}{2}} F\left(u^{\epsilon_{n}}\right), A^{\frac{1}{2}} v>_{\left[L^{2}(\Omega)\right]^{3}} d t \\
& \rightarrow \int_{0}^{T}<A^{-\frac{1}{2}} F(U), A^{\frac{1}{2}} v>_{\left[L^{2}(\Omega)\right]^{3}} d t
\end{aligned}
$$

due to the estimate

$$
\begin{aligned}
& \int_{0}^{T}\left\|A^{-\frac{1}{2}}\left[F\left(u^{\epsilon_{n}}\right)-F(U)\right]\right\|_{\left[L^{2}(\Omega)\right]^{3}} d t \leq \int_{0}^{T}\left\|\left|u^{\epsilon_{n}}-U\right|\left(\left|u^{\epsilon_{n}}\right|+|U|\right)\right\|_{\left[L^{2}(\Omega)\right]^{3}} d t \\
& \quad \leq \int_{0}^{T}\left\|u^{\epsilon_{n}}-U\right\|_{\left[L^{4}(\Omega)\right]^{3}}\left(\left\|u^{\epsilon_{n}}\right\|_{\left[L^{4}(\Omega)\right]^{3}}+\|U\|_{\left[L^{4}(\Omega)\right]^{3}}\right) d t \\
& \quad \leq \int_{0}^{T}\left\|u^{\epsilon_{n}}-U\right\|_{\left[H^{1^{-}}(\Omega)\right]^{3}}\left(\left\|u^{\epsilon_{n}}\right\|_{\left[H^{1^{-}}(\Omega)\right]^{3}}+\|U\|_{\left[H^{1^{-}}(\Omega)\right]^{3}}\right) d t \\
& \leq\left\|u^{\epsilon_{n}}-U\right\|_{L^{2}\left(0, T ;\left[H^{1^{-}}(\Omega)\right]^{3}\right)}\left(\left\|u^{\epsilon_{n}}\right\|_{L^{2}\left(0, T ;\left[H^{1^{-}}(\Omega)\right]^{3}\right)}\right. \\
& \left.\quad+\|U\|_{L^{2}\left(0, T ;\left[H^{1^{-}}(\Omega)\right]^{3}\right)}\right) \rightarrow 0, \quad \epsilon_{n} \rightarrow 0^{+} .
\end{aligned}
$$

Finally, thanks to [37, Lemma 1.1] and the convergence $u^{\epsilon_{n}} \rightarrow U$ in $L^{2}(0, T$; $\left.\left[H_{0}^{1^{-}}(\Omega)\right]^{3}\right)$ (or weak in $L^{2}\left(0, T ;\left[H_{0}^{1}(\Omega)\right]^{3}\right)$ ), we have

$$
<u_{t}^{\epsilon_{n}}, v>_{\left[L^{2}(\Omega)\right]^{3}}=\frac{d}{d t}<u^{\epsilon_{n}}, v>_{\left[L^{2}(\Omega)\right]^{3}} \rightarrow \frac{d}{d t}<U, v>_{\left[L^{2}(\Omega)\right]^{3}},
$$

in the sense of 'scalar distributions' (e.g. $[33,37])$; the derivative $\frac{d}{d t}$ in the sense of distributions. 
Consequently, we were able to pass to the limit in the weakly formulated regularization (2.10) of the 3-D N-S equation. We obtain

$$
\begin{aligned}
\frac{d}{d t}<U, v>_{\left[L^{2}(\Omega)\right]^{3}}= & -<A^{\frac{1}{2}} U, A^{\frac{1}{2}} v>_{\left[L^{2}(\Omega)\right]^{3}}+<A^{-\frac{1}{2}} F(U), A^{\frac{1}{2}} v>_{\left[L^{2}(\Omega)\right]^{3}} \\
& +<P f, v>_{\left[L^{2}(\Omega)\right]^{3}},
\end{aligned}
$$

where $v \in D\left(A^{\frac{1}{2}}\right)$ was arbitrary, and the convergence of the first right hand side component is in the weak sense. We obtain a weak solution to the 3-D N-S equation in the spirit of the Leray original definition (e.g. [40, p. 139]). Such solution is global in time, as was first observed in [24].

Note that the weak solution obtained above is global in time, while eventually not unique, since it depends on the chosen subsequence. For regular initial data

$u_{0} \in D\left(A_{2}^{\frac{3}{4}}\right)$ it must coincide (for small times) with the unique local strong solution described in Theorem 1.5, since the last exists on certain time interval $t \in[0, \tau)$ and fulfills (2.18) for $T<\tau$.

\section{2-D Critical N-S Equation as a Limit of Sub-Critical Approximations}

We will describe now the convergence of the solutions of the 2-D fractal approximations (1.2) to the solution of the limiting 2-D N-S equation. Precisely as in Theorem 1.6, the solutions $u^{\alpha}$ of (1.2) (the superscript is added for clarity) will be constructed in the class:

$u^{\alpha} \in C\left(\left([0, \tau) ; D\left({A^{\frac{1^{+}}{}}}^{+}\right)\right) \cap C\left((0, \tau) ; D\left(A^{\frac{3}{4}}\right)\right), u_{t}^{\alpha} \in C\left((0, \tau) ; D\left(A^{\frac{3}{4}^{-}}\right)\right)\right.$.

Further, the $L^{2}(\Omega)$ a priori estimates are satisfied for $u^{\alpha}$ uniformly in $\alpha \in\left(0, \frac{1}{2}\right]$. Consequently, we claim:

Theorem 3.1 The local in time solution $u^{\alpha}(t)$ of the approximating problem (1.2) constructed as in Theorem 1.6 will be extended globally in time in the above class. Moreover, the standard $L^{2}(\Omega)$ a priori estimate (1.11) is valid for it uniformly in $\alpha \in\left(0, \frac{1}{2}\right]$.

All the nice properties of the abstract semilinear sectorial equation, like elegant theory of existence and uniqueness, regularization of the solution for positive times, are valid for solutions $u^{\alpha}(t)$ with any $\alpha>0$. Typical regularization goes from the phase space $X^{\beta}, \beta<1$, to the space $X^{1}=D(A)$; see e.g. [3,21]. However, for problems (1.2), estimates of norms better that $L^{2}(\Omega)$ will depend on $\alpha>0$, possibly blowing up when $\alpha \rightarrow 0^{+}$, and therefore can not be extended in general to a limit solution $u$.

\subsection{2-D (1.1) as a Limit of (1.2) When $\alpha \rightarrow 0^{+}$}

A precise description of letting $\alpha \rightarrow 0^{+}$in the equation (1.2) is given next. In this section we consider the solutions $u^{\alpha}$ of (1.2) constructed in Theorem 3.1 on the 
phase space $D\left(A^{\frac{3}{4}}\right) \subset\left[H^{\frac{3}{2}}(\Omega)\right]^{2}$. Such solutions, for any $\alpha \in\left(0, \frac{1}{2}\right]$, are varying in $\left[H^{\frac{3}{2}}(\Omega)\right]^{2}$. Indeed, according to [20, Proposition 1.4]: For any $\beta \geq 0$, the domain $D\left(A^{\beta}\right)$ is continuously embedded in $X_{2} \cap\left[H^{2 \beta}(\Omega)\right]^{2}$. Solutions $u^{\alpha}$ fulfill also, uniformly in $\alpha \in\left(0, \frac{1}{2}\right]$, estimate (1.11) in $\left[L^{2}(\Omega)\right]^{2}$. More precisely, for such solutions of (1.2) we have an estimate:

$$
\exists_{\text {const }>0} \forall_{\alpha \in\left(0, \frac{1}{2}\right]}\left\|u^{\alpha}\right\|_{L^{\infty}\left([0, \infty) ;\left[L^{2}(\Omega)\right]^{2}\right)} \leq \text { const } .
$$

This is the main information allowing us to let $\alpha \rightarrow 0^{+}$in the equation (1.2).

Passing in (1.2) to the limit $\alpha \rightarrow 0^{+}$. We look at (1.2) as an equation in $\left[L^{2}(\Omega)\right]^{2}$, and 'multiply' by the test function $A^{-1-\alpha} \phi$ where $\phi \in D\left(A^{\frac{3}{4}}\right)$; recall that $D\left(A^{\frac{3}{4}}\right) \subset$ $\left[H^{\frac{3}{2}}(\Omega)\right]^{2}$,

$$
\begin{aligned}
<u_{t}^{\alpha}+P\left(u^{\alpha} \cdot \nabla\right) u^{\alpha}, A^{-1-\alpha} \phi>_{\left[L^{2}(\Omega)\right]^{2}=} & -<A^{1+\alpha} u^{\alpha}, A^{-1-\alpha} \phi>_{\left[L^{2}(\Omega)\right]^{2}} \\
& +<P f, A^{-1-\alpha} \phi>_{\left[L^{2}(\Omega)\right]^{2}} .
\end{aligned}
$$

We will discuss now the convergence of the terms in (3.3) one by one. Note that when $\alpha \rightarrow 0^{+}$then, by Lemma $4.1, A^{-1-\alpha} \phi \rightarrow A^{-1} \phi$. Thanks to uniform in $\alpha \in\left(0, \frac{1}{2}\right]$ boundedness of $u^{\alpha}$ in $L^{\infty}\left([0, \infty) ;\left[L^{2}(\Omega)\right]^{2}\right)$, we obtain:

$$
\begin{aligned}
& <A^{1+\alpha} u^{\alpha}, A^{-1-\alpha} \phi>_{\left[L^{2}(\Omega)\right]^{2}}=<u^{\alpha}, \phi>_{\left[L^{2}(\Omega)\right]^{2}} \rightarrow<u, \phi>_{\left[L^{2}(\Omega)\right]^{2}}, \\
& <P f, A^{-1-\alpha} \phi>_{\left[L^{2}(\Omega)\right]^{2}} \rightarrow<P f, A^{-1} \phi>_{\left[L^{2}(\Omega)\right]^{2}},
\end{aligned}
$$

where $u$ is the weak limit of $u^{\alpha}$ in $\left[L^{2}(\Omega)\right]^{2}$ as $\alpha \rightarrow 0^{+}$(over a sequence $\left\{\alpha_{n}\right\}$ convergent to $0^{+}$; various sequences may lead to various weak limits).

We return to (3.3) to see that letting $\alpha \rightarrow 0^{+}$over a sequence $\left\{\alpha_{n}\right\}$, where $u$ denotes weak limit in $\left[L^{2}(\Omega)\right]^{2}$ of such sequence, we have

$$
\begin{aligned}
\lim _{\alpha_{n} \rightarrow 0} & <A^{-\alpha}\left(u_{t}^{\alpha}+P\left(u^{\alpha} \cdot \nabla\right) u^{\alpha}\right), A^{-1} \phi>_{\left[L^{2}(\Omega)\right]^{2}} \\
& =-<u, \phi>_{\left[L^{2}(\Omega)\right]^{2}}+<P f, A^{-1} \phi>_{\left[L^{2}(\Omega)\right]^{2}},
\end{aligned}
$$

since the right hand side is convergent. Consequently, the left hand side has a limit as $\alpha_{n} \rightarrow 0^{+}$,

$$
\lim _{\alpha_{n} \rightarrow 0}<A^{-\alpha}\left(u_{t}^{\alpha}+P\left(u^{\alpha} \cdot \nabla\right) u^{\alpha}\right), A^{-1} \phi>_{\left[L^{2}(\Omega)\right]^{2}}=\omega_{\phi}
$$

Note that, for the 'test functions' $A^{-1} \phi$ varying in a separable Banach space, passing countable many times to a subsequence, we can chose a common subsequence proper for all test functions in dense subset of the space. Consequently, the equation below will be fulfilled in the whole space. We obtain

$$
\forall_{\phi \in X_{2}} \quad \omega_{\phi}=-<u, \phi>_{\left[L^{2}(\Omega)\right]^{2}}+<P f, A^{-1} \phi>_{\left[L^{2}(\Omega)\right]^{2}},
$$

which is a weak form of the limiting equation. 
Separation of terms The two terms of $\left[A^{-\alpha}\left(u_{t}^{\alpha}+P\left(u^{\alpha} \cdot \nabla\right) u^{\alpha}\right)\right]$ will be separated when letting $\alpha \rightarrow 0^{+}$. More precisely we have:

Remark 3.2 Since the approximating solutions $u^{\alpha}$ satisfy (in particular)

$$
u^{\alpha} \in L^{\infty}\left(0, T ;\left[L^{2}(\Omega)\right]^{2}\right), u_{t}^{\alpha} \in L^{2}\left(0, T ;\left[L^{2}(\Omega)\right]^{2}\right),
$$

then by [37, Lemma 1.1, Chapt. III]

$$
\forall_{\eta \in X_{2}}<u_{t}^{\alpha}, \eta>_{\left[L^{2}(\Omega)\right]^{2}}=\frac{d}{d t}<u^{\alpha}, \eta>_{\left[L^{2}(\Omega)\right]^{2}} \rightarrow \frac{d}{d t}<u, \eta>_{\left[L^{2}(\Omega)\right]^{2}},
$$

the time derivative $\frac{d}{d t}$ and the convergence are understood in $\mathcal{D}^{\prime}\left(0, T ;\left[L^{2}(\Omega)\right]^{2}\right)($ space of the 'scalar distributions' [33]). Consequently,

$$
\omega_{\phi}=\frac{d}{d t}<A^{-\alpha} u, A^{-1} \phi>_{\left[L^{2}(\Omega)\right]^{2}}+\omega_{\phi}^{1}
$$

where $\omega_{\phi}^{1}$ is a limit in $\mathcal{D}^{\prime}\left(0, T ;\left[L^{2}(\Omega)\right]^{2}\right)$ of $<P\left(u^{\alpha} \cdot \nabla\right) u^{\alpha}, A^{-1-\alpha} \phi>_{\left[L^{2}(\Omega)\right]^{2}}$ over a chosen sequence $\alpha_{n} \rightarrow 0^{+}$.

Convergence of the nonlinear term $F\left(u^{\alpha}\right)=P\left(u^{\alpha} \cdot \nabla\right) u^{\alpha}$ will be discussed next. As seen from (1.12), the approximating solutions $u^{\alpha}$ are bounded in $L^{2}\left(0, T ;\left[H_{0}^{1}(\Omega)\right]^{2}\right)$ uniformly in $\alpha>0$. Estimate (1.15):

$$
\left\|A^{-\frac{1}{2}} F\left(u^{\alpha}\right)\right\|_{\left[L^{2}(\Omega)\right]^{2}} \leq c\left\|u^{\alpha}\right\|_{\left[L^{4}(\Omega)\right]^{2}}^{2} \leq c^{\prime}\left\|u^{\alpha}\right\|_{\left[L^{2}(\Omega)\right]^{2}}\left\|u^{\alpha}\right\|_{\left[H_{0}^{1}(\Omega)\right]^{2}}, N=2,
$$

together with the consequence of Eq. (1.2)

$$
A^{-\left(\frac{1}{2}+\alpha\right)} u_{t}^{\alpha}=-A^{\frac{1}{2}} u^{\alpha}+A^{-\left(\frac{1}{2}+\alpha\right)} F\left(u^{\alpha}\right)+A^{-\left(\frac{1}{2}+\alpha\right)} P f \in L^{2}\left(0, T ;\left[L^{2}(\Omega)\right]^{2}\right),
$$

show that $u_{t}^{\alpha}$ are bounded in $L^{2}\left(0, T ; D\left(A^{-1}\right)\right)$ uniformly in $\alpha \in\left(0, \frac{1}{2}\right]$. By LionsAubin compactness lemma [33, Theorem 5.1], the family $\left\{u^{\alpha}\right\}_{\alpha \in\left(0, \frac{1}{2}\right]}$ as bounded in the space

$$
W=\left\{\phi ; \phi \in L^{2}\left(0, T ;\left[H_{0}^{1}(\Omega)\right]^{2}\right), \phi_{t} \in L^{2}\left(0, T ; D\left(A^{-1}\right)\right)\right\}
$$

is precompact in $L^{2}\left(0, T ;\left[H_{0}^{1^{-}}(\Omega)\right]^{2}\right)$. In particular any sequence $\left\{u^{\alpha_{n}}\right\}, \alpha_{n} \rightarrow 0^{+}$, has a subsequence convergent almost everywhere in $(0, T) \times \Omega$. Moreover, the above compactness allows to pass to a limit (using estimates of Observation 1.4) in the nonlinear term in (3.3). Indeed, if $u^{\alpha_{n}} \rightarrow u, \alpha_{n} \rightarrow 0^{+}$in the above sense then, using a consequence of [20, Lemma 2.2]; for small $\epsilon>0$

$$
\left\|A^{-\left(\frac{1}{2}+\epsilon\right)} P\left[(u \cdot \nabla) u-\left(u^{\alpha_{n}} \cdot \nabla\right) u^{\alpha_{n}}\right]\right\|_{\left[L^{2}(\Omega)\right]^{2}} \leq C\left\|\left|u-u^{\alpha_{n}}\right|\left(|u|+\left|u^{\alpha_{n}}\right|\right)\right\|_{\left[L^{s}(\Omega)\right]^{2}},
$$


where $\frac{1}{s}=\frac{1}{2}+\epsilon$ (so that $\left.s<2\right)$, we have

$$
\begin{aligned}
& \int_{0}^{T}<P\left(u^{\alpha_{n}} \cdot \nabla\right) u^{\alpha_{n}}, A^{-1-\alpha_{n}} \phi>_{\left[L^{2}(\Omega)\right]^{2}} d t \\
& =\int_{0}^{T}<A^{-\left(\frac{1}{2}+\epsilon\right)} P\left(u^{\alpha_{n}} \cdot \nabla\right) u^{\alpha_{n}}, A^{-\frac{1}{2}-\alpha_{n}+\epsilon} \phi>_{\left[L^{2}(\Omega)\right]^{2}} d t \\
& \rightarrow \int_{0}^{T}<A^{-\left(\frac{1}{2}+\epsilon\right)} P(u \cdot \nabla) u, A^{-\frac{1}{2}+\epsilon} \phi>_{\left[L^{2}(\Omega)\right]^{2}} d t
\end{aligned}
$$

The construction presented above allows us to formulate the following theorem:

Theorem 3.3 Let $\left\{u^{\alpha}\right\}_{\alpha \in\left(0, \frac{1}{2}\right]}$ be the set of regular $D\left(A^{\frac{3}{4}}\right)$ solutions to sub-critical equations (1.2). Such solutions are bounded in the space $\left[L^{2}(\Omega)\right]^{2}$, uniformly in $\alpha$. As a consequence of that and the regularity properties of such solutions (varying in $\left.D\left(A^{\frac{3}{4}}\right) \subset\left[H^{\frac{3}{2}}(\Omega)\right]^{2}\right)$, for arbitrary sequence $\left\{\alpha_{n}\right\} \subset\left(0, \frac{1}{2}\right]$ convergent to $0^{+}$we can find a subsequence $\left\{\alpha_{n_{k}}\right\}$ that the corresponding sequence of solutions $\left\{u^{\alpha_{n_{k}}}\right\}$ converges weakly in $\left[L^{2}(\Omega)\right]^{2}$ to a function u fulfilling the equation:

$$
\forall_{\phi \in X_{2}} \omega_{\phi}=-<u, \phi>_{\left[L^{2}(\Omega)\right]^{2}}+<P f, A^{-1} \phi>_{\left[L^{2}(\Omega)\right]^{2}}
$$

Due to denseness of the set $D\left(A^{\frac{3}{4}}\right)$ in $X_{2}$, the right hand side of (3.13) defines a unique element in $X_{2}$. The left hand side $\omega_{\phi}$ is defined in (3.6) and discussed in Remark 3.2.

Remark 3.4 As well known (e.g. [33, Theorem 6.2]), the $L^{\infty}\left(0, T ;\left[L^{2}(\Omega)\right]^{2}\right) \cap$ $L^{2}\left(0, T ; D\left(A^{\frac{1}{2}}\right)\right)$ solution of the 2-D N-S equation with $u_{t} \in L^{2}\left(0, T ; D\left(A^{-\frac{1}{2}}\right)\right)$ is unique. Indeed, if $u_{1}, u_{2}$ are two such (weak) solutions, applying projector $P$, taking the difference of the equations and multiplying the result by $w=u_{1}-u_{2}$, we get:

$$
\begin{aligned}
\frac{1}{2} \frac{d}{d t}\|w(t)\|_{\left[L^{2}(\Omega)\right]^{2}}^{2} \leq & -\left\|A^{\frac{1}{2}} w\right\|_{\left[L^{2}(\Omega)\right]^{2}}^{2}+<P(w \cdot \nabla) u_{1}, w>_{\left[L^{2}(\Omega)\right]^{2}} \\
& +<P\left(u_{2} \cdot \nabla\right) w, w>_{\left[L^{2}(\Omega)\right]^{2}} .
\end{aligned}
$$

The last term vanishes for divergence-free functions. The earlier term, using an equivalent form of the nonlinearity in (1.13) and the Nirenberg-Gagliardo inequality, is estimated as follows:

$$
\begin{aligned}
\left|<P(w \cdot \nabla) u_{1}, w>_{\left[L^{2}(\Omega)\right]^{2}}\right| & \leq c\|w\|_{\left[L^{4}(\Omega)\right]^{2}}^{2}\left\|A^{\frac{1}{2}} u_{1}\right\|_{\left[L^{2}(\Omega)\right]^{2}} \\
& \leq c\|w\|_{\left[L^{2}(\Omega)\right]^{2}}\left\|A^{\frac{1}{2}} w\right\|_{\left[L^{2}(\Omega)\right]^{2}}\left\|A^{\frac{1}{2}} u_{1}\right\|_{\left[L^{2}(\Omega)\right]^{2}}
\end{aligned}
$$


Inserting the last estimate into (3.14), using Cauchy's inequality, we obtain

$$
\begin{aligned}
\frac{1}{2} \frac{d}{d t}\|w(t)\|_{\left[L^{2}(\Omega)\right]^{2}}^{2} & \leq-\left\|A^{\frac{1}{2}} w\right\|_{\left[L^{2}(\Omega)\right]^{2}}^{2}+c\|w\|_{\left[L^{2}(\Omega)\right]^{2}}\left\|A^{\frac{1}{2}} w\right\|_{\left[L^{2}(\Omega)\right]^{2}}\left\|A^{\frac{1}{2}} u_{1}\right\|_{\left[L^{2}(\Omega)\right]^{2}} \\
& \leq C\|w\|_{\left[L^{2}(\Omega)\right]^{2}}^{2}\left\|A^{\frac{1}{2}} u_{1}\right\|_{\left[L^{2}(\Omega)\right]^{2}}^{2} .
\end{aligned}
$$

Since $\|w(0)\|_{\left[L^{2}(\Omega)\right]^{2}}^{2}=0$ then $\|w(t)\|_{\left[L^{2}(\Omega)\right]^{2}}^{2}=0$ for all $t \in[0, T]$, due to the Gronwall lemma.

Remark 3.5 In 2-D, convergence of the approximating solutions $u^{\alpha}$ of (1.2) to the unique solution $u$ of the N-S equation holds in a better sense whenever $u_{0} \in D\left(A^{\frac{1}{2}}\right)$. Multiplying (1.2) by $A u^{\alpha}$, we get

$$
\begin{aligned}
\frac{1}{2} \frac{d}{d t}\left\|A^{\frac{1}{2}} u^{\alpha}\right\|_{\left[L^{2}(\Omega)\right]^{2}}^{2}= & -\left\|A^{1+\frac{\alpha}{2}} u^{\alpha}\right\|_{\left[L^{2}(\Omega)\right]^{2}}^{2}+<P\left(u^{\alpha} \cdot \nabla\right) u^{\alpha}, A u^{\alpha}>_{\left[L^{2}(\Omega)\right]^{2}} \\
& +<P f, A u^{\alpha}>_{\left[L^{2}(\Omega)\right]^{2}} .
\end{aligned}
$$

Further, due to (1.16), Nirenberg-Gagliardo and Young inequalities,

$$
\begin{aligned}
\mid & <P\left(u^{\alpha} \cdot \nabla\right) u^{\alpha}, A u^{\alpha}>_{\left[L^{2}(\Omega)\right]^{2}} \mid \\
& \leq\left\|A^{-\frac{\alpha}{2}} P\left(u^{\alpha} \cdot \nabla\right) u^{\alpha}\right\|_{\left[L^{2}(\Omega)\right]^{2}}\left\|A^{1+\frac{\alpha}{2}} u^{\alpha}\right\|_{\left[L^{2}(\Omega)\right]^{2}} \\
& \leq c\left\|u^{\alpha}\right\|_{\left[L^{4}(\Omega)\right]^{2}}^{1+\alpha}\left\|\nabla u^{\alpha}\right\|_{\left[L^{4}(\Omega)\right]^{2}}^{1-\alpha}\left\|A^{1+\frac{\alpha}{2}} u^{\alpha}\right\|_{\left[L^{2}(\Omega)\right]^{2}} \\
& \leq \epsilon\left\|A^{1+\frac{\alpha}{2}} u^{\alpha}\right\|_{\left[L^{2}(\Omega)\right]^{2}}^{2}+C_{\epsilon}\left\|u^{\alpha}\right\|_{\left[H^{1}(\Omega)\right]^{2}}^{\frac{2\left[2+3 \alpha+\alpha^{2}\right]}{1+5 \alpha}}\left\|u^{\alpha}\right\|_{\left[L^{2}(\Omega)\right]^{2}}^{\frac{2\left[3+4 \alpha-\alpha^{2}\right]}{1+5 \alpha}} .
\end{aligned}
$$

For small $\epsilon>0, \alpha>0$, we thus have

$$
\begin{aligned}
\frac{1}{2} \frac{d}{d t}\left\|A^{\frac{1}{2}} u^{\alpha}\right\|_{\left[L^{2}(\Omega)\right]^{2}}^{2} \leq & -\frac{1}{4}\left\|A^{1+\frac{\alpha}{2}} u^{\alpha}\right\|_{\left[L^{2}(\Omega)\right]^{2}}^{2}+C_{\epsilon}\left\|u^{\alpha}\right\|_{\left[H^{1}(\Omega)\right]^{2}}^{\frac{2\left[2+3 \alpha+\alpha^{2}\right]}{1+5 \alpha}}\left\|u^{\alpha}\right\|_{\left[L^{2}(\Omega)\right]^{2}}^{\frac{2\left[3+4 \alpha-\alpha^{2}\right]}{1+5 \alpha}} \\
& +\|P f\|_{\left[L^{2}(\Omega)\right]^{2}}^{2}
\end{aligned}
$$

note that $\frac{2\left[2+3 \alpha+\alpha^{2}\right]}{1+5 \alpha}<4$. It follows from the natural a priori estimates and Gronwall inequality that the family $\left\{u^{\alpha}\right\}_{\alpha \in\left(0, \frac{1}{2}\right]}$ is bounded in the norm

$$
L^{\infty}\left(0, T ;\left[H_{0}^{1}(\Omega)\right]^{2}\right) \cap L^{2}(0, T ; D(A)) .
$$

Consequently, it is precompact in

$$
L^{p}\left(0, T ;\left[H_{0}^{1^{-}}(\Omega)\right]^{2}\right) \cap L^{2}\left(0, T ; D\left(A^{1^{-}}\right)\right),
$$

with arbitrary $p>1$. The convergence $u^{\alpha} \rightarrow u$ as $\alpha \rightarrow 0^{+}$is thus verified in the above space. 
Remark 3.6 To understand better the method used in the study of global extendibility of solutions we will discuss shortly an example ${ }^{1}$ of the Burgers type system in 3-D, obtained by neglecting the viscosity in homogeneous 3-D N-S equation:

$$
\begin{aligned}
& U_{t}=v \Delta U-(U \cdot \nabla) U, \quad x \in \Omega, t>0 \\
& U=0, \quad t>0, \quad x \in \partial \Omega \\
& U(0, x)=U_{0}(x)
\end{aligned}
$$

It is easy to see that each component of the sufficiently regular (that is, varying in $\left.\left[L^{\infty}(\Omega)\right]^{3}\right)$ solution of (3.20) fulfills Maximum Principle:

$$
\left\|U_{i}(t, \cdot)\right\|_{L^{\infty}(\Omega)} \leq\left\|U_{0 i}\right\|_{L^{\infty}(\Omega)}, \quad i=1,2,3 .
$$

We are thus given a natural a priori estimate in $Y=\left[L^{\infty}(\Omega)\right]^{3}$ for such system. The nonlinear term is as in the N-S equation, and we have the estimates:

$$
\begin{aligned}
\|(U \cdot \nabla) U\|_{\left[L^{2}(\Omega)\right]^{3}} & \leq\|U\|_{\left[L^{\infty}(\Omega)\right]^{3}}\|U\|_{\left[H_{0}^{1}(\Omega)\right]^{3}} \leq\left\|U_{0}\right\|_{\left[L^{\infty}(\Omega)\right]^{3}}\|U\|_{\left[H_{0}^{1}(\Omega)\right]^{3}}, \\
\|(U \cdot \nabla) U\|_{\left[H^{-1}(\Omega)\right]^{3}} & \leq c\|U\|_{\left[L^{\infty}(\Omega)\right]^{3}}^{2},
\end{aligned}
$$

valid in particular for all the local solutions varying in $\left[H^{\frac{3}{2}^{+}}(\Omega)\right]^{3} \cap\left[H_{0}^{1}(\Omega)\right]^{3}$ (as in Theorem 1.5). Since $H^{3^{2}}(\Omega) \subset L^{\infty}(\Omega)$, the Maximum Principle works for such solutions. By interpolation, (3.22) gives

$$
\|(U \cdot \nabla) U\|_{\left[H^{\left.-\frac{1}{4}(\Omega)\right]^{3}}\right.} \leq c\|U\|_{\left[L^{\infty}(\Omega)\right]^{3}}^{\frac{5}{4}}\|U\|_{\left[H_{0}^{1}(\Omega)\right]^{3}}^{\frac{3}{4}} \leq c\left\|U_{0}\right\|_{\left[L^{\infty}(\Omega)\right]^{3}}^{\frac{5}{4}}\|U\|_{\left[H_{0}^{1}(\Omega)\right]^{3}}^{\frac{3}{4}},
$$

which shows the nonlinearity is sub-critical in that case. Consequently, the local solution will be extended globally in time.

The above example shows that our approach is sensitive (through subordination condition (1.8)) not only on the form of nonlinearity, but also another specific properties of the problem. It also indicates the role of the pressure in the classical N-S equation, the term which seems responsible for the delicate properties of solutions of that equation.

Remark 3.7 It is evident from the considerations above that the phenomenon of loosing regularity by local smooth solutions of 3-D N-S is possible only if they enter the supercritical range of the nonlinearity (compare (1.28)). It would be therefore interesting to consider local solutions corresponding to initial data $u_{0}$ fulfilling

$$
\left\|A^{-\frac{1}{8}} F\left(u_{0}\right)\right\|_{\left[L^{2}(\Omega)\right]^{3}} \geq g\left(\left\|u_{0}\right\|_{\left[L^{2}(\Omega)\right]^{3}}\right)\left\|A^{\frac{7}{8}} u_{0}\right\|_{\left[L^{2}(\Omega)\right]^{3}}^{1+\varepsilon},
$$

where $0<\varepsilon<\frac{2}{7}$, as eventual candidates for such phenomenon.

\footnotetext{
${ }^{1}$ Suggested kindly by professor Eduard Feireisl.
} 


\section{Conclusion}

The PROBLEM connected with the 3-D Navier-Stokes equation is not solved in the paper, while we are explaining its reason. The viscosity in the classical N-S equation together with the standard a priori estimate could not be used in general to prevent better norms of its solutions from blowing-up in a finite time, especially in 3-D case. In order, for small initial data we enjoy the standard property of problems with quadratic nonlinearity, that near zero function the estimates of nonlinearity obtained from the equation are proportional to the square of the norm, therefore there is a ball centered at zero the solutions originated in it will never leave that ball. For such solutions, the nonlinear term in N-S is subordinated to the main part operator. Of course, if a better estimate was known, for example the estimate in $L^{N}(\Omega)\left(L^{N^{+}}(\Omega)\right)$, it would make the N-D Navier-Stokes equation critical (sub-critical) due to the following calculation

$$
\begin{aligned}
\|P(u \cdot \nabla) u\|_{X^{-\frac{1}{2}}} & \leq c\left\||u|^{2}\right\|_{\left[L^{2}(\Omega)\right]^{N}} \leq c^{\prime}\|u\|_{\left[L^{\left.\frac{2 N}{N-2}(\Omega)\right]^{N}}\right.}\|u\|_{\left[L^{N}(\Omega)\right]^{N}} \\
& \leq c^{\prime \prime}\|u\|_{X^{\frac{1}{2}}}\|u\|_{\left[L^{N}(\Omega)\right]^{N}} .
\end{aligned}
$$

An alternative possibility is mentioned in Remark 3.7.

It seems that the fractal regularizations; (1.2) in 2-D and (2.6), $s>\frac{5}{4}$, in 3-D, having much better properties of solutions, offer an alternative way of description of the flow and for numerical calculations where, for small values of parameters, solutions of the regularizations are globally existing and regular approximations of solutions to the original N-S equation.

Acknowledgements The paper is dedicated to my mentors, who teach me through the years 1980-90. The author was supported by NCN Grant DEC-2012/05/B/ST1/00546 (Poland). He want also to thank the Referee for valuable remarks improving the original version of the manuscript.

Open Access This article is distributed under the terms of the Creative Commons Attribution 4.0 International License (http://creativecommons.org/licenses/by/4.0/), which permits unrestricted use, distribution, and reproduction in any medium, provided you give appropriate credit to the original author(s) and the source, provide a link to the Creative Commons license, and indicate if changes were made.

\section{Appendix: Fractional Powers Operators and Estimates}

\section{Some Technicalities}

When passing to the limit in the considerations above it was important that the estimates, in particular the constants in it, can be taken uniform in $\alpha$. Therefore, in the technical lemmas below we need to care on a very precise expression of that uniformity. Even some estimates can be found in the literature, usually such uniformity is not clear from the presentation, thus we include here the proofs for completeness.

First, we formulate a lemma used in the previous calculations: 
Lemma 4.1 Let $A$ be a positive operator in a Banach space $X([3,34,39])$. For arbitrary $\phi \in X$, we have

$$
\forall_{\epsilon>0} \exists L\left\|\left(I-A^{-\beta}\right) \phi\right\|_{X} \leq \sin (\pi \beta)\left(\frac{2 L(1+M)}{\pi}+L^{-1} M\right)\|\phi\|_{X}+\epsilon .
$$

Consequently, the left hand side tends to zero as $0<\beta \rightarrow 0^{+}$.

Proof Our task is, for fixed $\phi \in X$ and $\beta$ near $0^{+}$, to estimate the expression:

$$
\begin{aligned}
\left(A^{-\beta}-I\right) \phi & =\frac{\sin (\pi \beta)}{\pi} \int_{0}^{\infty} \lambda^{-\beta}(\lambda+A)^{-1} \phi d \lambda-\frac{\sin (\pi(1-\beta))}{\pi} \int_{0}^{\infty} \frac{\lambda^{(1-\beta)-1}}{\lambda+1} d \lambda \phi \\
& =\frac{\sin (\pi \beta)}{\pi} \int_{0}^{\infty} \lambda^{-\beta}\left[(\lambda+A)^{-1} \phi-\frac{1}{\lambda+1} \phi\right] d \lambda .
\end{aligned}
$$

In the estimates we are using the following properties; taken from [34, p. 62] equality valid for $\eta \in(0,1)$

$$
\int_{0}^{\infty} \frac{\lambda^{\eta-1}}{\lambda+1} d \lambda=\frac{\pi}{\sin (\pi \eta)}
$$

the simple formula:

$$
(\lambda+A)^{-1} \phi-\frac{1}{\lambda+1} \phi=\frac{1}{\lambda+1}\left[\lambda(\lambda+A)^{-1} \phi-\phi+(\lambda+A)^{-1} \phi\right],
$$

and the two asymptotic properties of non-negative operators valid on functions $\phi \in X$ taken from [34, Proposition 1.1.3]:

$$
\begin{aligned}
& \lim _{\lambda \rightarrow \infty} \lambda(\lambda+A)^{-1} \phi=\phi, \\
& \lim _{\lambda \rightarrow \infty}(\lambda+A)^{-1} A \phi=0 .
\end{aligned}
$$

Returning to the proof, we split the integral in $(4.2)$ into $(0, L)$ and $(L, \infty)$ and estimate the first part,

$$
\begin{aligned}
& \frac{\sin (\pi \beta)}{\pi}\left\|\int_{0}^{L} \lambda^{-\beta}\left(\frac{1}{\lambda+1}-(\lambda+A)^{-1}\right) \phi d \lambda\right\|_{X} \\
& \leq \frac{\sin (\pi \beta)}{\pi} \int_{0}^{L} \lambda^{-\beta}(1+M) d \lambda\|\phi\|_{X} \\
& =\frac{\sin (\pi \beta)}{\pi} \frac{L^{1-\beta}}{1-\beta}(1+M)\|\phi\|_{X},
\end{aligned}
$$

where $L>0$ will be chosen later. Note that letting $\beta \rightarrow 0^{+}$the result of the estimate above is bounded by $\left|\frac{\sin (\pi \beta)}{\pi}\right| 2 L(1+M)\|\phi\|_{X} \rightarrow 0$, for any fixed $L>0$. 
Next using (4.3), the integral over $(L, \infty)$ is, for $\phi \in X$, estimated as follows:

$$
\begin{aligned}
& \frac{\sin (\pi \beta)}{\pi}\left\|\int_{L}^{\infty} \lambda^{-\beta}\left[(\lambda+A)^{-1} \phi-\frac{1}{\lambda+1} \phi\right] d \lambda\right\|_{X} \\
& \leq \frac{\sin (\pi \beta)}{\pi} \int_{L}^{\infty} \frac{\lambda^{-\beta}}{\lambda+1}\left\|\lambda(\lambda+A)^{-1} \phi-\phi+(\lambda+A)^{-1} \phi\right\|_{X} d \lambda,
\end{aligned}
$$

where due to (4.4) we see that

$$
\begin{gathered}
\left\|(\lambda+1)(\lambda+A)^{-1} A \phi-\phi\right\|_{X} \leq \\
\left\|\lambda(\lambda+A)^{-1} \phi-\phi\right\|_{X}+\left\|(\lambda+A)^{-1} \phi\right\|_{X} \leq \epsilon \\
+\frac{M}{1+\lambda}\|\phi\|_{X} \text { as } \lambda \rightarrow \infty
\end{gathered}
$$

$\epsilon>0$ arbitrary fixed. Consequently we obtain:

$$
\begin{aligned}
& \frac{\sin (\pi \beta)}{\pi} \int_{L}^{\infty} \frac{\lambda^{-\beta}}{\lambda+1}\left(\epsilon+\frac{M}{1+\lambda}\|\phi\|_{X}\right) d \lambda \\
& \leq \frac{\sin (\pi \beta)}{\pi} \int_{L}^{\infty} \lambda^{-\beta-1}\left(\epsilon+\frac{M}{\lambda}\|\phi\|_{X}\right) d \lambda \\
& \leq \frac{\sin (\pi \beta)}{\pi} \frac{L^{-\beta}}{\beta} \epsilon+\frac{\sin (\pi \beta)}{\pi} \frac{L^{-\beta-1}}{\beta+1} M\|\phi\|_{X},
\end{aligned}
$$

for sufficiently large value of $L \geq 1$, as specified in (4.7). Note that letting $\beta \rightarrow 0^{+}$ in the resulting estimate we have:

$$
\frac{\sin (\pi \beta)}{\pi \beta} L^{-\beta} \epsilon+\frac{\sin (\pi \beta)}{\pi} \frac{L^{-\beta-1}}{\beta+1} M\|\phi\|_{X} \leq \epsilon+\sin (\pi \beta) L^{-1} M\|\phi\|_{X},
$$

for chosen large value of $L$.

For such $L$ we get a final estimate of the integral in (4.2) having the form:

$$
\begin{aligned}
\left\|\left(A^{-\beta}-I\right) \phi\right\|_{X} & \leq \frac{\sin (\pi \beta)}{\pi}\left\|\int_{0}^{\infty} \lambda^{-\beta}\left[(\lambda+A)^{-1} \phi-\frac{1}{\lambda+1} \phi\right] d \lambda\right\|_{X} \\
& \leq \sin (\pi \beta)\left(\frac{2 L(1+M)}{\pi}+L^{-1} M\right)\|\phi\|_{X}+\epsilon
\end{aligned}
$$

where $\epsilon>0$ was arbitrary. The right hand side of (4.10) will be made small when we let $\beta$ near $0^{+}$, noting $\epsilon$ was an arbitrary positive number.

\section{Properties of the Fractional Powers Operators}

Recall first the Balakrishnan definition of fractional power of non-negative operator (e.g. [27, p. 299], [28]). Let $A$ be a closed linear densely defined operator in a Banach 
space $X$, such that its resolvent set contains $(-\infty, 0)$ and the resolvent satisfies:

$$
\left\|\lambda(\lambda+A)^{-1}\right\| \leq M, \lambda>0
$$

Then, for $\eta \in(0,1)$,

$$
A^{\eta} \phi=\frac{\sin (\pi \eta)}{\pi} \int_{0}^{\infty} s^{\eta-1} A(s+A)^{-1} \phi d s .
$$

Note that there is another definition, through singular integrals, of the fractional powers of the $(-\Delta)^{-\alpha}$ in $L^{p}\left(\mathbb{R}^{N}\right)$ frequently used in the literature. See [34, Chapter 2.2] for the proof of equivalence of the two definitions for $1<p<\frac{N}{2 \Re \alpha}$; see also [11, section 4.3]. See further [32] for the commutator estimates.

Moment inequality We recall here, the moment inequality estimate valid for fractional powers of non-negative operators. They are suitable to compare various fractional powers. Recalling [46, p. 98], we have the following version of the moment inequality with precise constant; for $0 \leq \alpha<\beta<\gamma \leq 1$,

$$
\left\|A^{\beta} \phi\right\| \leq \frac{\left(\sin \frac{\pi(\beta-\alpha)}{\gamma-\alpha}\right)(\gamma-\alpha)^{2}}{\pi(\gamma-\beta)(\beta-\alpha)}(M+1)\left\|A^{\gamma} \phi\right\|^{\frac{\beta-\alpha}{\gamma-\alpha}}\left\|A^{\alpha} \phi\right\|^{\frac{\gamma-\beta}{\gamma-\alpha}},
$$

where $\phi \in D\left(A^{\gamma}\right)$.

\section{Moment Inequality Extended}

The task here is to extend the moment inequality to the form suitable to compare the powers $1+\alpha$ and 1 (here $\alpha>0$ near $0^{+}$). We need to use a more general than (4.11) expression (1.6) for the fractional powers, taken from [34, (3.4), p. 59], which states that:

$$
A^{1+\alpha} \phi=\frac{2 \sin (\pi \alpha)}{(1-\alpha) \pi} \int_{0}^{\infty} \lambda^{\alpha}\left[A(\lambda+A)^{-1}\right]^{2} \phi d \lambda, \quad \phi \in D\left(A^{2}\right),
$$

where the original term $\frac{\Gamma(2)}{\Gamma(\alpha) \Gamma(2-\alpha)}$ has been transformed using the known properties of the $\Gamma$ function;

$$
\Gamma(1+\alpha)=\alpha \Gamma(\alpha), \quad \Gamma(\alpha) \Gamma(1-\alpha)=\frac{\pi}{\sin (\pi \alpha)}, \quad \Gamma(2)=2
$$

We are using the following bound, valid for positive operators, in the calculations below:

$$
\left\|A(\lambda+A)^{-1}\right\|_{X} \leq M+1
$$


splitting the integral over $(0, L)$ and $(L, \infty)$, we estimate the first integral,

$$
\begin{aligned}
& \frac{2 \sin (\pi \alpha)}{(1-\alpha) \pi} \int_{0}^{L} \lambda^{\alpha}\left[A(\lambda+A)^{-1}\right]^{2} \phi d \lambda \\
& =\frac{2 \sin (\pi \alpha)}{(1-\alpha) \pi} \int_{0}^{L} \lambda^{\alpha}(\lambda+A)^{-1}\left[A(\lambda+A)^{-1}\right] A \phi d \lambda \\
& =\frac{2 \sin (\pi \alpha)}{(1-\alpha) \pi} \int_{0}^{L} \lambda^{\alpha} \frac{M}{1+\lambda}(M+1) d \lambda\|A \phi\|_{X} .
\end{aligned}
$$

We thus have:

$$
\left\|\frac{2 \sin (\pi \alpha)}{(1-\alpha) \pi} \int_{0}^{L} \lambda^{\alpha}\left[A(\lambda+A)^{-1}\right]^{2} \phi d \lambda\right\|_{X} \leq \frac{2 \sin (\pi \alpha)}{(1-\alpha) \pi} M(M+1) \frac{L^{\alpha}}{\alpha}\|A \phi\|_{X} .
$$

The second integral over $(L, \infty)$ is estimated next:

$$
\begin{aligned}
\frac{2 \sin (\pi \alpha)}{(1-\alpha) \pi}\left\|\int_{L}^{\infty} \lambda^{\alpha}\left[(\lambda+A)^{-1}\right]^{2} A^{2} \phi d \lambda\right\|_{X} & \leq \frac{2 \sin (\pi \alpha)}{(1-\alpha) \pi} \int_{L}^{\infty} \lambda^{\alpha}\left(\frac{M}{\lambda}\right)^{2}\left\|A^{2} \phi\right\|_{X} d \lambda \\
& =\frac{2 \sin (\pi \alpha)}{(1-\alpha) \pi} \frac{M^{2}}{1-\alpha} L^{\alpha-1}\left\|A^{2} \phi\right\|_{X} .
\end{aligned}
$$

Minimizing with respect to $L>0$ we get $L_{\text {min }}=\frac{M\left\|A^{2} \phi\right\|_{X}}{(M+1)\|A \phi\|_{X}}$, which leads to the final estimate:

$$
\begin{aligned}
\left\|A^{1+\alpha} \phi\right\|_{X} & \leq \frac{2 \sin (\pi \alpha)}{(1-\alpha) \pi} M(M+1) \frac{L_{\text {min }}^{\alpha}}{\alpha}\|A \phi\|_{X}+\frac{2 \sin (\pi \alpha)}{(1-\alpha) \pi} \frac{M^{2}}{1-\alpha} L_{\text {min }}^{\alpha-1}\left\|A^{2} \phi\right\|_{X} \\
& =\frac{2 \sin (\pi \alpha)}{(1-\alpha)^{2} \pi \alpha} M^{1+\alpha}(M+1)^{\alpha}\|A \phi\|_{X}^{1-\alpha}\left\|A^{2} \phi\right\|_{X}^{\alpha} \rightarrow 2 M\|A \phi\|_{X},
\end{aligned}
$$

as $\alpha \rightarrow 0^{+}$.

\section{References}

1. Amann, H.: Linear and Quasilinear Parabolic Problems, Volume I, Abstract Linear Theory. Birkhaüser Verlag, Basel (1995)

2. Cholewa, J.W., Dlotko, T.: Local attractor for $n-D$ Navier-Stokes system. Hiroshima Math. J. 28, 309-319 (1998)

3. Cholewa, J.W., Dlotko, T.: Global Attractors in Abstract Parabolic Problems. Cambridge University Press, Cambridge (2000)

4. Constantin, P., Cordoba, D., Wu, J.: On the critical dissipative Quasi-geostrophic equation. Indiana Univ. Math. J. 50, 97-108 (2001) 
5. Cordoba, A., Cordoba, D.: A Maximum Principle applied to quasi-geostrophic equations. Commun. Math. Phys. 249, 511-528 (2004)

6. Cordoba, A., Cordoba, D.: A pointwise estimate for fractionary derivatives with applications to partial differential equations. Proc. Natl. Acad. Sci. 100, 15316-15317 (2003)

7. Dlotko, T.: The generalized Korteweg-de Vries-Burgers equation in $H^{2}(\mathbb{R})$. Nonlinear Anal. TMA 74, 721-732 (2011)

8. Dlotko, T., Sun, C.: Dynamics of the modified viscous Cahn-Hilliard equation in $\mathbb{R}^{N}$. Topol. Methods Nonlinear Anal. 35, 277-294 (2010)

9. Dlotko, T., Kania, M.B., Sun, C.: Analysis of the viscous Cahn-Hilliard equation in $\mathbb{R}^{N}$. J. Differ. Equ. 252, 2771-2791 (2012)

10. Dlotko, T., Kania, M.B.: Subcritical Hamilton-Jacobi fractional equation in $\mathbb{R}^{N}$. Math. Methods Appl. Sci. 38, 2547-2560 (2015)

11. Dlotko, T., Kania, M.B., Sun, C.: Quasi-geostrophic equation in $R^{2}$. J. Differ. Equ. 259, 531-561 (2015)

12. Dlotko, T., Kania, M.B., Ma, S.: Korteweg-de Vries-Burgers system in $\mathbb{R}^{N}$. J. Math. Anal. Appl. 411, 853-872 (2014)

13. Dlotko, T., Kania, M.B., Yang, M.: Generalized Korteweg-de Vries equation in $H^{1}(R)$. Nonlinear Anal. TMA 71, 3934-3947 (2009)

14. Foias, C., Holm, D.D., Titi, E.S.: The Navier-alpha model of fluid turbulence. Phys. D Nonlinear Phenom. 152-153, 505-519 (2001)

15. Foias, C., Manley, O., Rosa, R., Temam, R.: Navier-Stokes equations and turbulence, Encyclopedia of Mathematics and its applications. Cambridge University Press, Cambridge (2004)

16. Fujita, H., Kato, T.: On the Navier-Stokes initial value problem. I. Arch. Ration. Mach. Anal. 16, 269-315 (1964)

17. Giga, Y.: Analyticity of the semigroup generated by the Stokes operator in $L_{r}$ spaces. Math. Z. 178, 297-329 (1981)

18. Giga, Y.: Domains of fractional powers of the Stokes operator in $L_{r}$ spaces. Arch. Ration. Mech. Anal. 89, 251-265 (1985)

19. Giga, Y.: Weak and strong solutions of the Navier-Stokes initial value problem. Publ. Res. Inst. Math. Sci. 19, 887-910 (1983)

20. Giga, Y., Miyakawa, T.: Solutions in $L_{r}$ of the Navier-Stokes initial value problem. Arch. Ration. Mech. Anal. 89, 267-281 (1985)

21. Henry, D.: Geometric Theory of Semilinear Parabolic Equations. Springer-Verlag, Berlin (1981)

22. Henry, D.: How to remember the Sobolev inequalities. In: de Figueiredo, D.G., Hönig, C.S. (eds.) Differential equations, pp. 97-109. Springer-Verlag, New York (1982)

23. Heywood, J.G.: The Navier-Stokes equations; on the existence, regularity and decay of solutions. Indiana Univ. Math. J. 29, 639-681 (1980)

24. Hopf, E.: Über die Anfangswertaufgabe für die hydrodynamischen Grundgleichungen. Math. Nachr. 4, 213-231 (1951)

25. Kato, T., Fujita, H.: On the nonstationary Navier-Stokes system. Rend. Sem. Mat. Univ. Padova 32, 243-260 (1962)

26. Kiselev, A., Nazarov, F., Volberg, A.: Global well-posedness for the critical $2 D$ dissipative quasigeostrophic equation. Invent. Math. 167, 445-453 (2007)

27. Komatsu, H.: Fractional powers of operators. Pacific J. Math. 19, 285-346 (1966)

28. Komatsu, H.: Fractional powers of operators, II. Interpolation spaces. Pacific J. Math. 21, 89-111 (1967)

29. Ladyzhenskaya, O.A.: The Mathematical Theory of Viscous Incompressible Flow, Revised English edition, Gordon and Breach (1969)

30. Ladyzhenskaya, O.A.: On some gaps in two of my papers on the Navier-Stokes equations and the way of closing them. J. Math. Sci. 115, 2789-2891 (2003)

31. Leray, J.: Essai sur le mouvement d'un fluide visqueux emplissant l'espace. Acta Math. 63, 193-248 (1934)

32. Linares, F., Ponce, G.: Introduction to Nonlinear Dispersive Equations. IMPA, Rio de Janeiro (2008)

33. Lions, J.-L.: Quelques méthodes de résolution des problè mes aux limites non linéaires. Dunod Gauthier-Villars, Paris (1969)

34. Martínez Carracedo, C., Sanz Alix, M.: The Theory of Fractional Powers of Operators. Elsevier, Amsterdam (2001) 
35. Rodriguez-Bernal, A.: Existence, uniqueness and regularity of solutions of nonlinear evolution equations in extended scales of Hilbert spaces, CDSNS91-61 Report. Georgia Institute of Technology, Atlanta (1991)

36. Sobolevskii, P.E.: On non-stationary equations of hydrodynamics for viscous fluid. Dokl. Akad. Nauk SSSR 128, 45-48 (1959). (in Russian)

37. Temam, R.: Navier-Stokes Equations, Theory and Numerical Analysis, North-Holland, Amsterdam (1979)

38. Temam, R.: On the Euler equations of incompressible perfect fluids. J. Funct. Anal. 20, 32-43 (1975)

39. Triebel, H.: Interpolation Theory, Function Spaces, Differential Operators. Veb Deutscher Verlag, Berlin (1978)

40. von Wahl, W.: Equations of Navier-Stokes and Abstract Parabolic Equations. Vieweg, Braunschweig/Wiesbaden (1985)

41. von Wahl, W.: Global solutions to evolution equations of parabolic type. In: Favini, A., Obrecht, E. (eds.) Differential Equations in Banach Spaces, Proceedings, 1985, Lecture Notes in Mathematics, vol. 1223, pp. 254-266. Springer, Berlin (1986)

42. Walter, W.: Differential and Integral Inequalities. Springer-Verlag, New York (1970)

43. Weissler, F.B.: The Navier-Stokes initial value problem in $L^{p}$. Arch. Ration. Mach. Anal. 74, 219-230 (1980)

44. Wu, J.: Dissipative quasi-geostrophic equations with $L^{p}$ data. Electron. J. Differ. Equ. 56, 1-13 (2001)

45. Wu, J.: The quasi-geostrophic equation and its two regularizations. Commun. Partial Differ. Equ. 27, 1161-1181 (2002)

46. Yagi, A.: Abstract Parabolic Evolution Equations and their Applications. Springer-Verlag, Heidelberg (2010) 